Research Article

\title{
NCF1/2/4 Are Prognostic Biomarkers Related to the Immune Infiltration of Kidney Renal Clear Cell Carcinoma
}

\author{
Yifei Chen, ${ }^{1}$ Fei He, ${ }^{2}$ Ruhua Wang, ${ }^{1}$ Menglin Yao, ${ }^{1}$ Yarui Li, ${ }^{1}$ Dan Guo, ${ }^{1}$ \\ and Shuixiang $\mathrm{He} \mathbb{1}^{1}$ \\ ${ }^{1}$ Department of Gastroenterology, First Affiliated Hospital of Xi'an Jiaotong University, Shaanxi 710061, China \\ ${ }^{2}$ Department of Urology, The First Affiliated Hospital of Xi'an Jiaotong University, Xi'an, Shaanxi 710061, China \\ Correspondence should be addressed to Shuixiang He; dyyyjxk@xjtu.edu.cn
}

Received 22 June 2021; Accepted 24 September 2021; Published 18 October 2021

Academic Editor: Sercan Ergün

Copyright (c) 2021 Yifei Chen et al. This is an open access article distributed under the Creative Commons Attribution License, which permits unrestricted use, distribution, and reproduction in any medium, provided the original work is properly cited.

\begin{abstract}
Neutrophil cytoplasmic factor 1/2/4 (NCF1/2/4) belongs to the NADPH oxidase complex, which is a cytoplasmic component, and its polymorphism is the main factor related to autoimmune diseases, which is probably caused by the regulation of peroxide. They also play a role in tumor growth and metastasis. This research is aimed at evaluating the biological function and prognostic role of NCF1, NCF2, and NCF4 genes in kidney renal clear cell carcinoma (KIRC) by using multiple online bioinformatics website, including Oncomine, GEPIA, UALCAN, Kaplan-Meier Plotter, TIMER, TISIDB, cBioPortal, LinkedOmics, GeneMANIA, and DAVID databases. The mRNA levels of NCFs were higher in KIRC tissues than in normal tissues. The overexpression of NCFs was significantly correlated with advanced pathological grades and individual cancer stages in KIRC. Meanwhile, the expressions of NCFs played an important role in the tumorigenesis and progression of KIRC. Prognostic value analysis suggested that high transcription levels of NCF1/4 were associated with poor overall survival in KIRC patients. In addition, results from the LinkedOmics database showed that the KEGG pathway related to NCFs mainly focused on immune activation and immune regulation function. NCF genetic alterations, including copy number amplification, missense mutation, and deep deletion, could be found through the cBioPortal database. Further, NCF expression was significantly correlated with infiltration levels of various immune cells as well as immune signatures. Protein-protein interaction network and enrichment analysis of NCF1/2/4 in KIRC showed that NCF coexpressed genes mainly associated with diverse immune marker sets showed significance. Overall, these results indicated that NCFs could be prognostic biomarkers as well as effective targets for diagnosis in KIRC.
\end{abstract}

\section{Introduction}

In recent years, the incidence of malignant kidney tumors has been increasing. In 2019, approximately 73,820 Americans were diagnosed with kidney cancer [1], and nearly 15,000 died of the disease $[2,3]$. It is the seventh most common cancer among men and the ninth most common cancer among women [4]. Kidney cancer represents several different types of cancer, which have different histology, clinical course, and response to treatment [5]. Clear cell carcinoma is the most common histological type of kidney cancer (75\%) [6] and one of the most aggressive types [7]. Because the kidney is located deep in the body, its clinical symptoms usually appear in the late stage, so the 3-year survival rate in the kidney renal clear cell carcinoma (KIRC) in the population is less than 5\% [8]. Until recently, we still lacked effective systemic therapies for KIRC, and surgery was the main treatment method [9]. In addition, the prognosis of KIRC is not good. One-third of patients will have local or distant metastases, and about one-quarter of patients undergoing radical surgery have recurred tumors at a distance [10]. Due to the high morbidity, high mortality, and difficulty of early diagnosis of KIRC, it is important to evaluate carcinogenic mechanisms and explore potential drug targets and molecular markers that have a prognostic value that affects the immune response of KIRC patients.

Neutrophil cytoplasmic factor 1 (NCF1), neutrophil cytoplasmic factor 2 (NCF2), and neutrophil cytoplasmic 
factor 4 (NCF4) are also referred to as p47phox, p67phox, and $\mathrm{p} 40$ phox, respectively. They belong to the NADPH oxidase complex, which is a cytoplasmic component, and its polymorphism is the main factor related to autoimmune diseases, which is probably caused by the regulation of peroxide [11]. In the case of inflammatory stimulation, NADPH oxidase is activated; phosphorylation of NCF1 (p47phox) leads to the assembly of NCF2 (p67phox) and NCF4 (p40phox) into an active oxidase complex p22phox/gp91phox. In this process, it promotes the conversion of oxygen $\left(\mathrm{O}_{2}\right)$ into superoxide ions $\left(\mathrm{O}_{2}^{-}\right)$and hydrogen peroxide $\left(\mathrm{H}_{2} \mathrm{O}_{2}\right)$, which are all reactive oxygen species (ROS) [12]. Studies have confirmed the correlation of these genes with chronic granulomatous disease [13], Crohn's disease [14], and autoimmune arthritis [15]. They also play a role in tumor growth and metastasis [16], such as Hodgkin's lymphoma [17]. However, their relationship with tumors in many other fields has not been explored, so this article is aimed at evaluating the biological function and prognostic role of NCF1, NCF2, and NCF4 genes in KIRC.

\section{Materials and Methods}

2.1. Oncomine Database Analysis. The mRNA levels of NCFs in various cancers were identified in the Oncomine database. The Oncomine database (http://www.oncomine.org) is an online cancer microarray database and synthetic genewide data-mining platform [18]. We compared the transcriptional levels of NCFs in different cancer tissues with their corresponding adjacent normal controls from the Oncomine database, using Student's $t$-test to generate a $p$ value. Cutoffs of $p$ value and fold change were defined as 0.05 and 2, respectively.

2.2. Gene Expression Profiling Interactive Analysis. Gene Expression Profiling Interactive Analysis (GEPIA) (http:// gepia.cancer-pku.cn/) integrates a tremendous amount of tumor and nontumor samples from The Cancer Genome Atlas (TCGA) and the Genotype-Tissue Expression (GTEx) database, providing differential expression analysis, correlation analysis, and patient survival analysis online [19]. In our study, GEPIA was used to analyze the expression of NCFs in kidney renal clear cell carcinoma with corresponding breast tissues. The cutoff of $p$ value was 0.05 and $\log _{2} \mathrm{FC}$ was 1 (fold change was 2). We also obtained the top 100 similar expression protein-coding genes with certain NCFs in KIRC Tumor dataset by similar gene module for Gene Ontology (GO) and Kyoto Encyclopedia of Genes and Genomes (KEGG) analysis.

2.3. UALCAN Database Analysis. UALCAN (http://ualcan .path.uab.edu) is a comprehensive web resource based on TCGA database level 3 RNA-seq and clinical data from 31 cancer types [20]. It can be used to estimate the relative transcriptional expression of query genes between tumor and normal samples as well as relative clinicopathologic parameters on patient survival. In this study, UALCAN was used to analyze the association between mRNA expressions of NCFs grouped by known prognostic factors (individual cancer stages, tumor grade, and KIRC subtype) in kidney renal clear cell carcinoma.

2.4. Kaplan-Meier Plotter Database. The research on the NCF prognostic value was performed in the KM-plotter database, which could evaluate the survival of more than 50,000 genes in 21 cancer types [21]. The ccRCC dataset with 530 samples was selected to explore the expression profile of NCFs on ccRCC overall survival (OS). Furthermore, the hazard ratio (HR), log-rank $p$ value, and survival plots were computed and output by the website automatically.

2.5. LinkedOmics Database Analysis. LinkedOmics is a publicly available portal that included multiomics data from 32 TCGA cancer types [22]. The "Linkfinder" module was used to perform the volcano map showing the NCF association results and heat map showing the coexpressed genes of NCFs. The "LinkInterpreter" module was used to perform GO and KEGG analysis of NCFs based on gene set enrichment analysis (GSEA). The criterion about GSEA is as follows: the minimum number of genes (size) is 3 and a simulation is 500 . The top 5 terms of GO and KEGG analysis were exhibited. Gene terms with $p$ value $<0.05$ and false discovery rate $($ FDR $)<0.05$ were considered significant.

2.6. cBioPortal Data Analysis. The cBioPortal for Cancer Genomics is a comprehensive web resource that can visualize and analyze multidimensional cancer genomic data [23]. Copy number variation (CNV), mutations, and the clinic outcomes of the gene types in KIRC were evaluated according to the online tools of cBioPortal. The $p$ value set as 0.05 was considered significantly different.

2.7. TIMER Database Analysis. The TIMER database is a comprehensive resource for systematical analysis of immune infiltrates across diverse cancer types [24]. Associations between NCF expression and TIIC infiltration levels were analyzed via TIMER, a website tool for analysis of genespecific correlation with TIICs. TIICs included B cells, $\mathrm{CD}^{+} \mathrm{T}$ cell, $\mathrm{CD}^{+} \mathrm{T}$ cell, macrophages, neutrophils, and dendritic cells.

2.8. TISIDB Database Analysis. Through the TISIDB online platform (http://cis.hku.hk/TISIDB/), the expression levels of NCFs in the six immune subtypes were analyzed [25].

2.9. Protein-Protein Interaction Network Construction. The STRING (http://string-db.org, version 11.0) database was used to predict the PPI network of DEGs and analyze the interactions between proteins [26]. GeneMANIA is an interactive and visual online protein-protein interaction (PPI) prediction tool, which provides the customizable function of the detection of genes with similar functions. GeneMANIA is a prediction website tool for analyzing genetic and protein interactions, coexpression, pathways, colocalization, and domain-protein similarity of target genes [27]. In this study, we analyzed the relationship between NCFs and their interactive genes by the GeneMANIA database and the STRING database. 
2.10. DAVID Database. DAVID contains a comprehensive set of functional annotation tools for better clarifying the biological functions of target genes [28]. In this work, Gene Ontology (GO) and Kyoto Encyclopedia of Genes and Genomes (KEGG) pathway enrichment analyses of NCFs and their coexpressed genes (100) were conducted using the DAVID tool. The cutoff value for significant GO terms and KEGG pathways was a false discover rate (FDR) of $<0.05$.

2.11. Cell Culture and Transfection. We purchased human normal renal tubular epithelial cell line HK-2 and kidney cancer cell line 786-O from the Cell Bank of Type Culture Collection of Shanghai Institute of Cell Biology. The cells were cultivated aseptically within DMEM medium (Gibco, San Diego, CA) that contained $10 \%$ fetal bovine serum (FBS) and maintained at $37^{\circ} \mathrm{C}$ in a humidified incubator with $5 \% \mathrm{CO}_{2}$. For $\mathrm{H}_{2} \mathrm{O}_{2}$-induced oxidative stress, $500 \mu \mathrm{M} \mathrm{H}_{2} \mathrm{O}_{2}$ was added to the culture medium. Small interfering RNA of targeted NCF4 RNA (si-NCF4, 50 nM) were designed and synthesized by RiboBio (China). Cell transfection was performed with the usage of Lipofectamine 2000 (Invitrogen), according to the protocol provided by the supplier.

2.12. Determination of ROS Formation. ROS formation was measured using $\mathrm{H}_{2}$ DCFDA (Invitrogen), after pretreatment with $4 \mu \mathrm{mol} \mathrm{H}_{2}$ DCFDA in serum-free and $\mathrm{pH}$ indicator-free medium for $30 \mathrm{~min}$. Then, incubation cells were washed twice with PBS and analyzed (FACSCalibur, Becton Dickinson). Relative ROS level was determined by fluorescence intensity.

2.13. Cell Counting Kit-8 (CCK-8) and Colony Formation. The Cell Counting Kit-8 (Beyotime Biotechnology, Jiangsu, China) was used to measure cell proliferation according to the manufacturer's protocol. Cells were seeded into 96-well plates at a density of $1 \times 10^{3}$ cells per well and determined every $24 \mathrm{~h}$. The way to determine was by adding $10 \mu \mathrm{l}$ of CCK-8 solution into each well and then culturing for 4 hours in an incubator. Four hours later, the absorbance value $(A)$ of cells was detected at the wavelength of $490 \mathrm{~nm}$. For colony formation assays, cells were seeded into six-well plates at the density of 500/well and were cultivated continuously at $37^{\circ} \mathrm{C}$ for $14 \mathrm{~d}$. Then, the colonies were washed and stained with $0.2 \%$ crystal violet solution for 2 hours.

2.14. Wound Healing and Transwell Assays. When the cell density attained $90 \%$ after 24 hours of transfection in 6well plates, the wound was scratched by a $10 \mu \mathrm{l}$ sterile tip and then washed off the floated cells. Under the microscope, photographs of the wound were taken at 0 and 36 hours. For transwell migration assays, $1 \times 10^{5}$ cells were plated into the upper chambers of a transwell apparatus (24-well insert, $8 \mu \mathrm{m}$ pore size, Corning) with $200 \mu \mathrm{l}$ of serum-free medium, and the bottom chamber was filled with $700 \mu \mathrm{l}$ medium supplemented with $10 \%$ FBS. After $24 \mathrm{~h}$ of incubation, the cells on the lower surface of the membrane were stained, photographed, and counted in six random fields per group using a microscope.
2.15. RNA Extraction and Western Blotting. According to the manufacturer's instructions, the total RNA was extracted from cultured cells by using Trizol reagent (Invitrogen, USA). Then, the cDNAs were synthesized following the protocol of PrimeScript ${ }^{\mathrm{TM}}$ RT Master Mix (Takara). Quantitative real-time PCR was conducted using SYBR Premix Ex Taq ${ }^{\mathrm{TM}}$ II (Takara) on Thermal Cycler CFX6 System (Bio-Rad). The relative transcriptional levels of target genes were calculated by using the $2-\Delta \Delta \mathrm{Ct}$ method. The RT-qPCR primer sequences were as follows: NCF1 forward primer $5^{\prime}$-ACGA GAGTGGTTGGTGGTTC- $3^{\prime}$ and reverse primer $5^{\prime}$-TGTA GGCTTTGATGGTGACG-3', NCF2 forward primer $5^{\prime}$ GCGCTAGGCTGGGACCTTGAAGCC- ${ }^{\prime}$ and reverse primer $5^{\prime}$-GTCTTGAAGAAGGGCAGTGATAAC- ${ }^{\prime}$, and NCF4 forward primer $5^{\prime}$-TGAACAGCTTCCGGAT GATG- $3^{\prime}$ and reverse primer $5^{\prime}$-TGAAGCCTCTCTTCTC CTCGAT- $3^{\prime}$. Western blot was performed by adding lysis buffer into cells and total protein was extracted. Equal amounts of protein samples were added to SDS/PAGE for conducting electrophoresis. Next, after being blocked for 1 hour with $5 \%$ defatted milk, the membranes with proteins were incubated in a primary antibody against NCF4 (Invitrogen), E-cadherin, $\alpha$-catenin, vimentin, MMP9, and $\beta$-actin (Cell Signaling Technology), overnight at $4^{\circ} \mathrm{C}$. Then, the membranes were incubated with the secondary antibodies and the proteins in the membranes were visualized by ECL after washing with TBST. $\beta$-Actin was used as a reference.

2.16. Statistical Analysis. Statistical analyses were performed using the SPSS 23.0 statistical software (IBM, USA), GraphPad Prism software (7.0). The quantitative data were shown as mean \pm standard deviation (SD) and were compared through Student's $t$-test. The enumeration data enlisted as percentages were compared via the chi-square test. It would be considered as statistical significance when $p<0.05$.

\section{Result}

3.1. Aberrant Expression of NCF1, NCF2, and NCF4 in Multiple Cancer Types and In-Depth Verification in KIRC. In order to preliminarily evaluate the role in tumorigenesis, we analyzed the different expression levels for NCF1, $\mathrm{NCF} 2$, and NCF4 between tumor and adjacent normal tissues in all TCGA tumors (Figure 1(a)). Our results showed that the levels of NCF expression are significantly downregulated in multiple cancer types including LUAD (lung adenocarcinoma) and LUSC (lung squamous cell carcinoma), but were higher in GBM (glioblastoma multiforme), KIRC (kidney renal clear cell carcinoma), and KIRP (kidney renal papillary cell carcinoma). These data show that NCF1, NCF2, and NCF4 have different expression levels in different cancers, suggesting that NCFs exerted diverse functions in various cancers. Differential expression was observed between tumor and normal tissues for NCF1, NCF2, and NCF4 in KIRC data from TCGA. The results indicated that NCF1, NCF2, and NCF4 were upregulated in KIRC compared with adjacent normal tissues $(p<0.001$; 

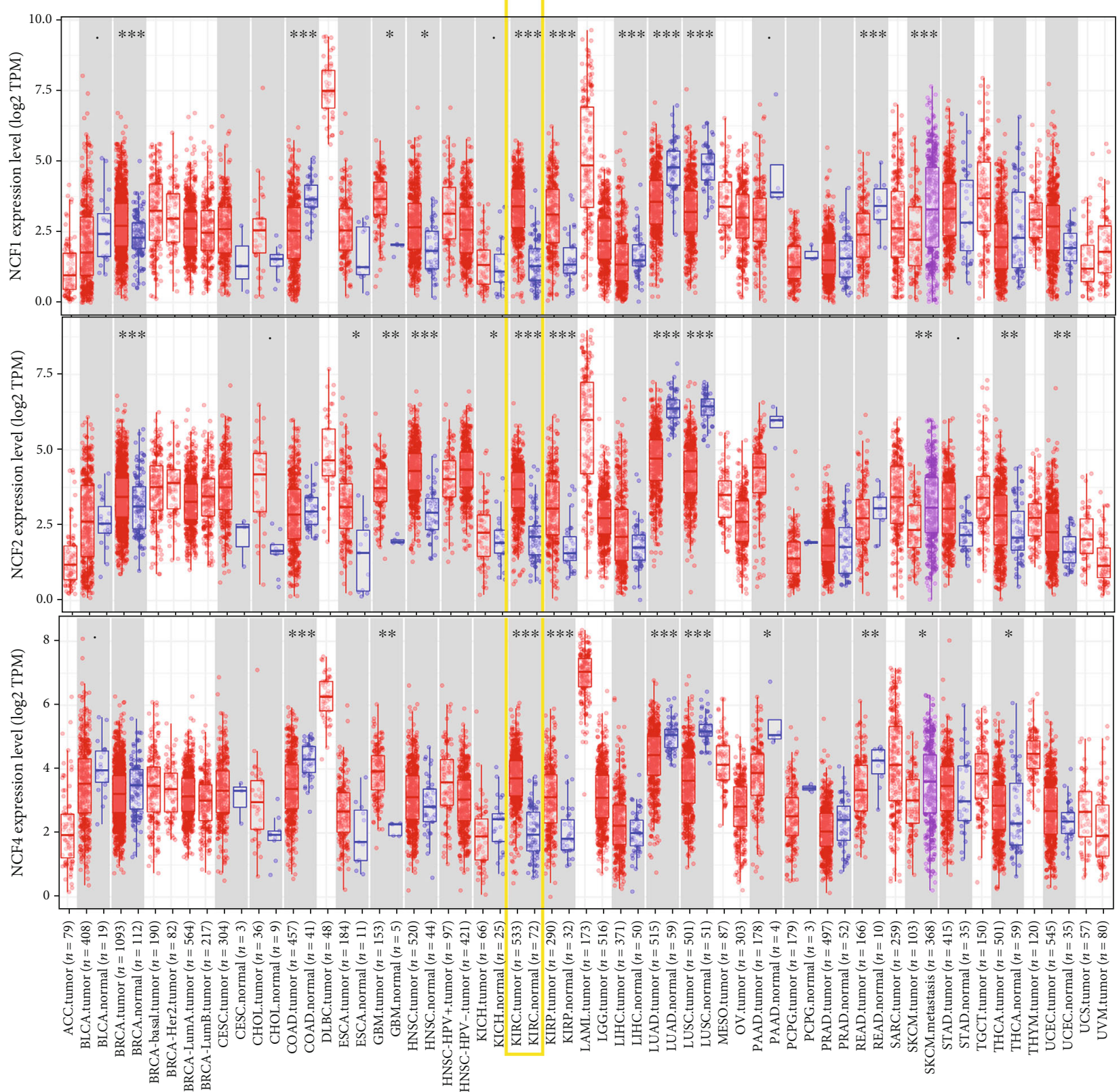

(a)

Figure 1: Continued. 


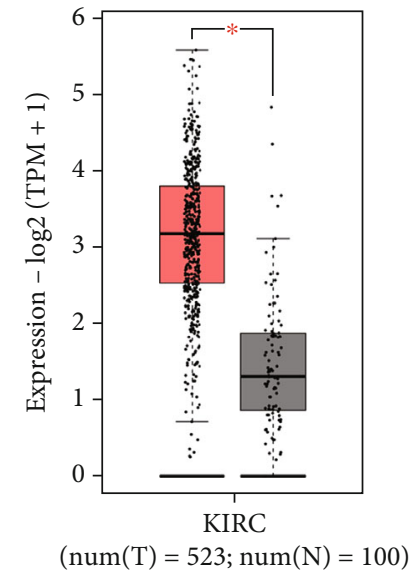

(b)

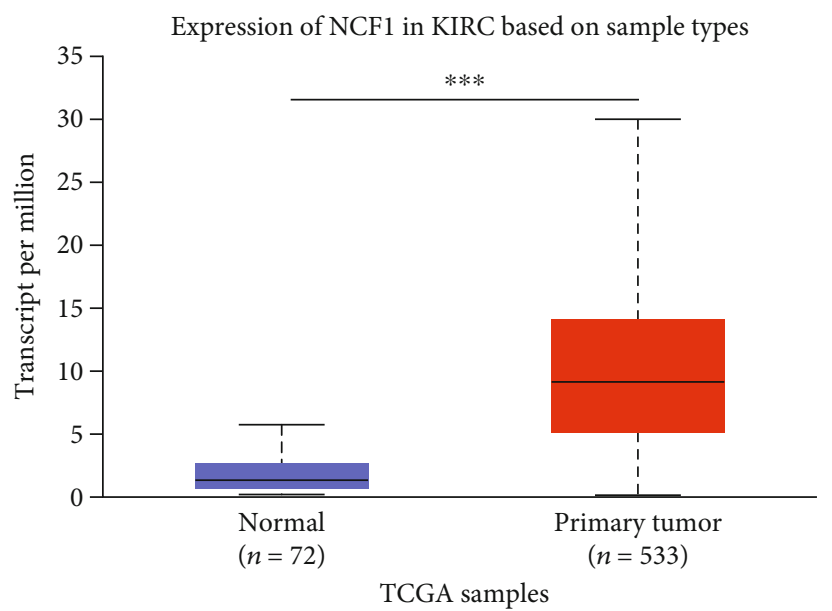

(e)

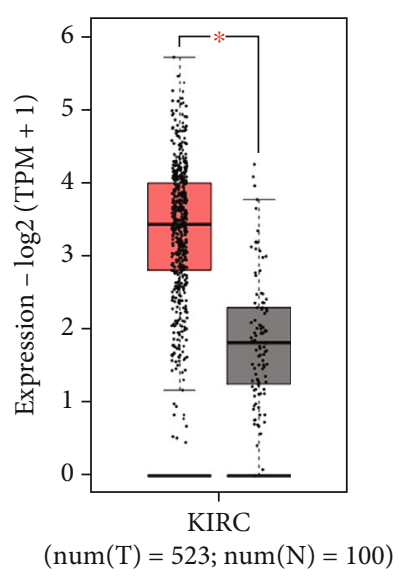

(c)

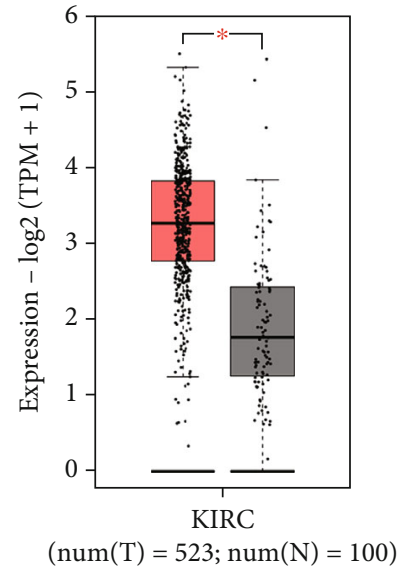

(d)

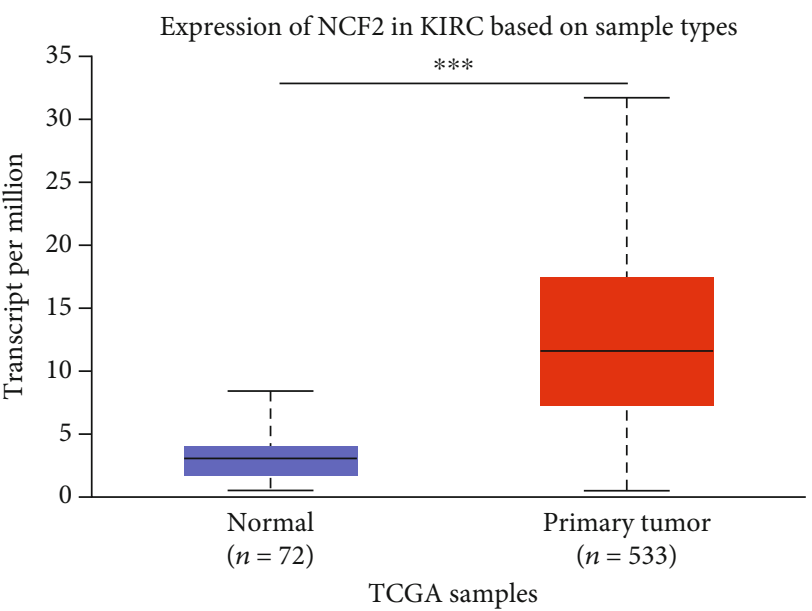

(f)

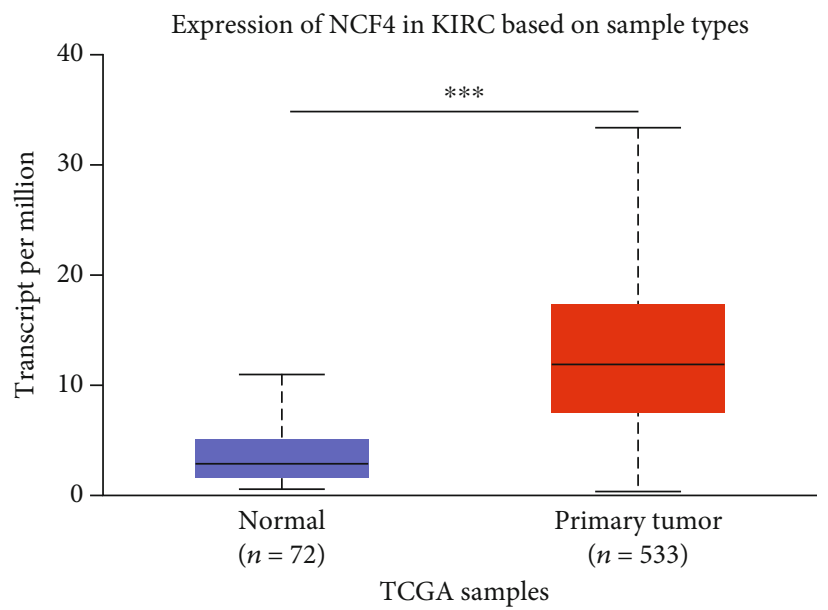

(g)

FIGURE 1: The expression levels of NCF1, NCF2, and NCF4 in KIRC were higher than those in the respective adjacent normal tissues. (a) Expression levels of NCF1, NCF2, and NCF4 in different types of tumors from TCGA database were determined using TIMER 2.0. (b-d) Expression levels of NCF1, NCF2, and NCF4 in KIRC tissues compared with normal tissues using the GEPIA database. (e, f) Expression levels of NCF1, NCF2, and NCF4 were expressed as box plots in normal individuals and patients with KIRC using the UALCAN database. ${ }^{*} p<0.05,{ }^{* *} p<0.01$, and ${ }^{* * *} p<0.001$. 
Expression of NCF1 in KIRC based on tumor grade

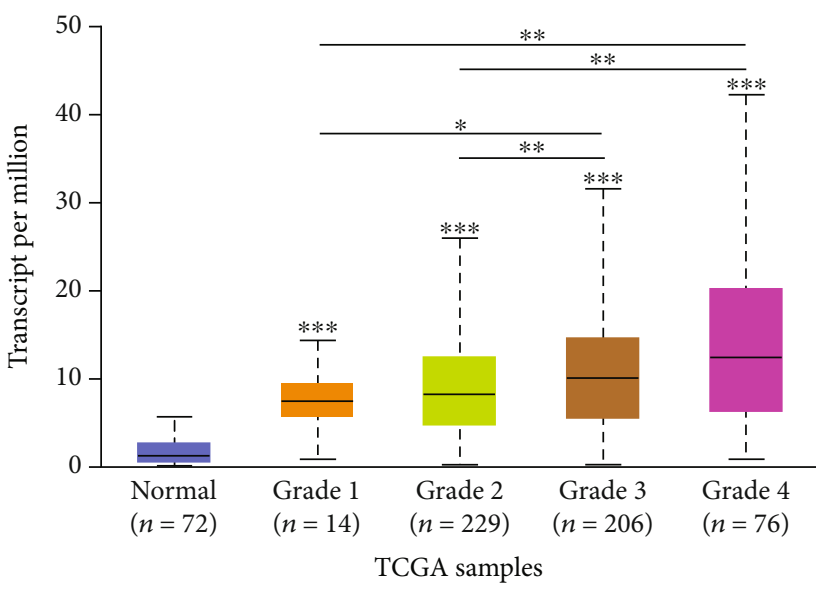

(a)

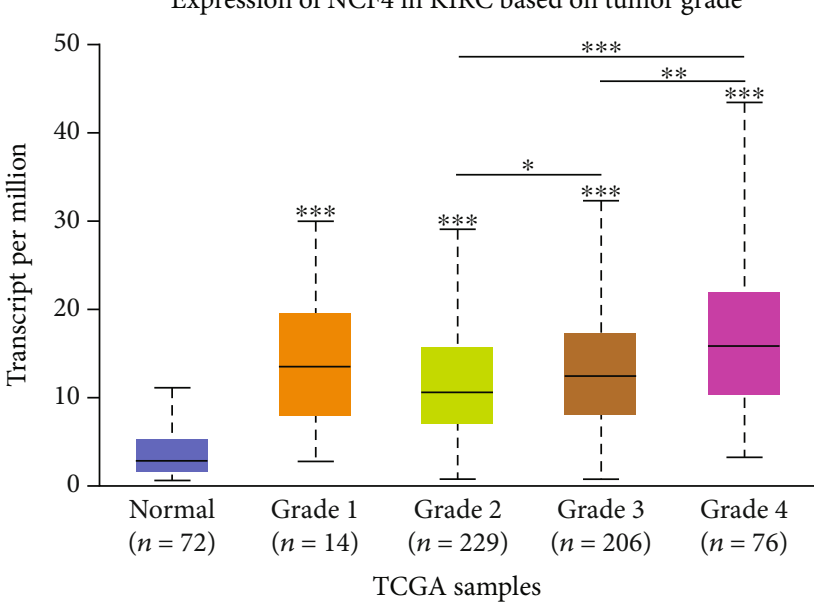

(c)

Expression of NCF2 in KIRC based on individual cancer stages

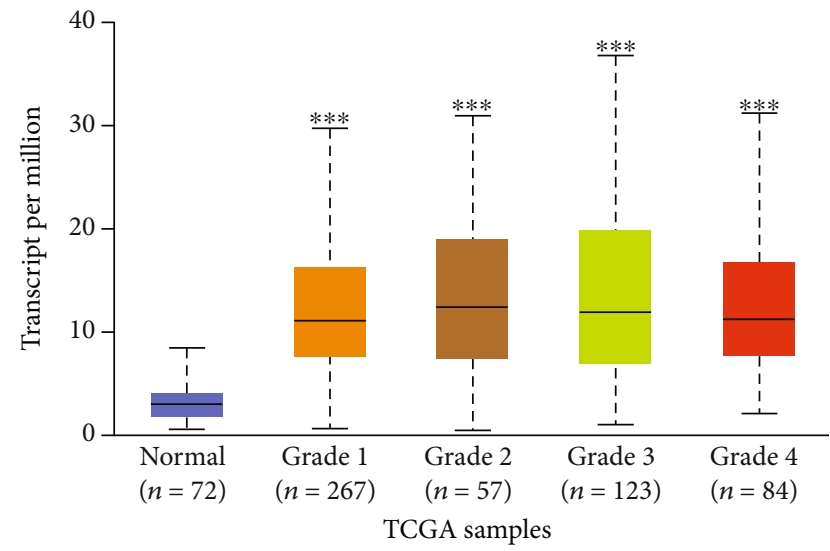

(e)
Expression of NCF2 in KIRC based on tumor grade

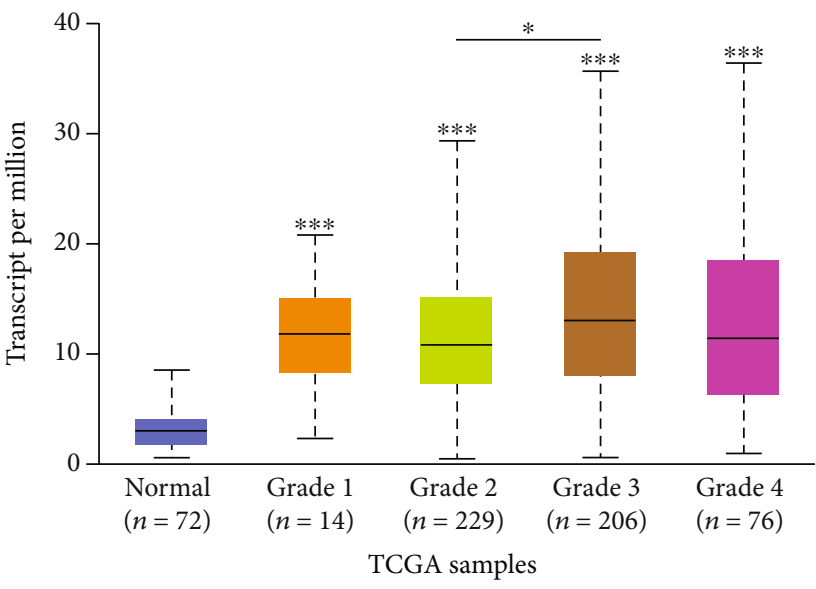

(b)

Expression of NCF1 in KIRC based on individual cancer stages

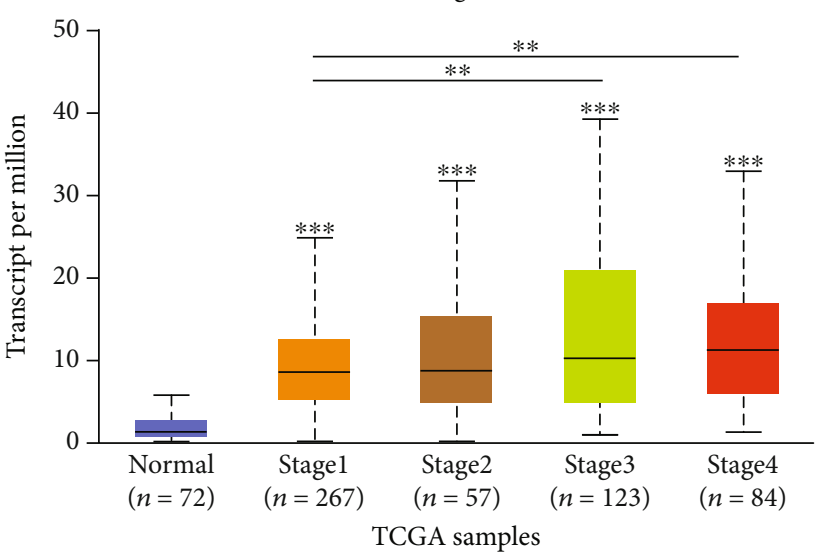

(d)

Expression of NCF4 in KIRC based on individual cancer stages

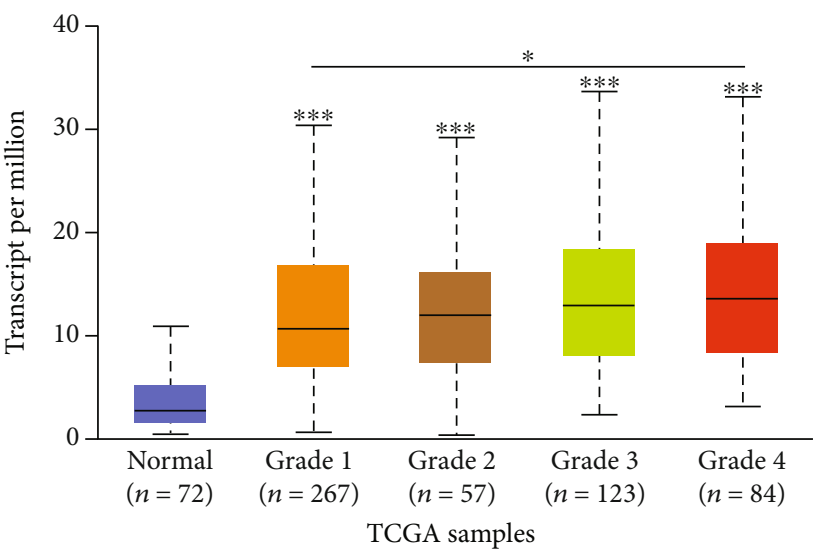

(f)

Figure 2: Continued. 


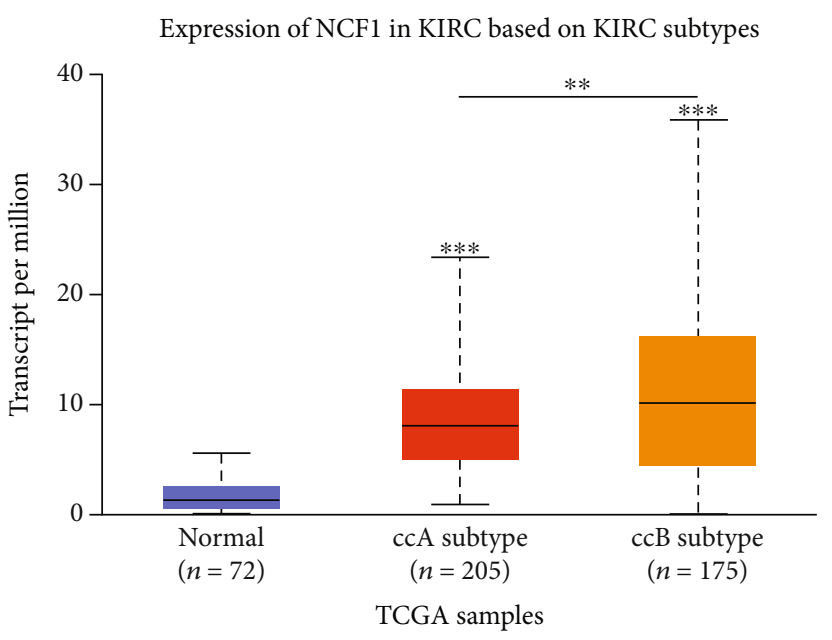

(g)

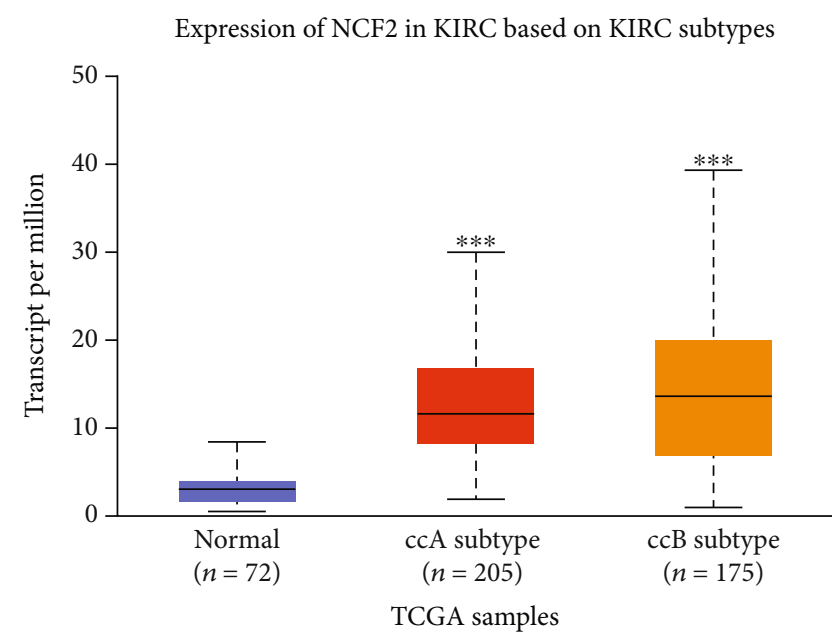

(h)

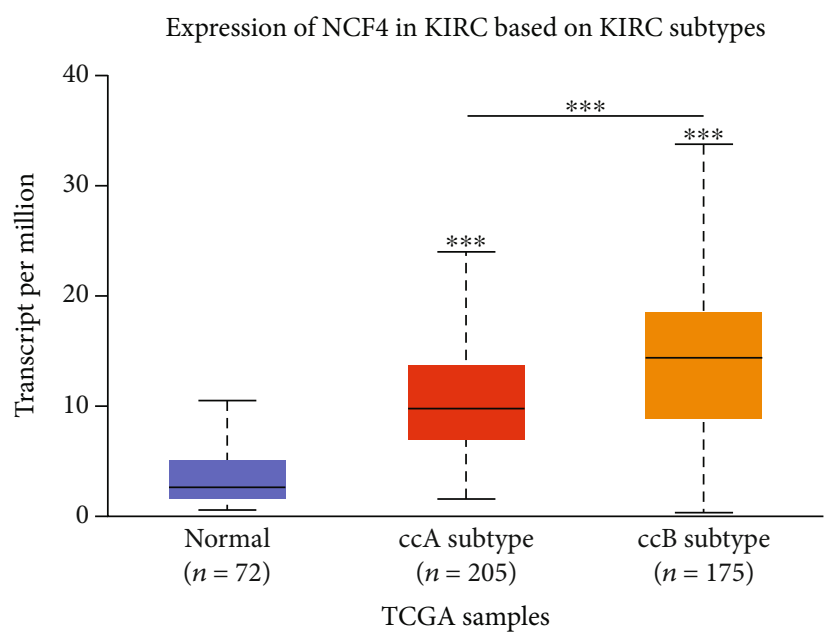

(i)

FIGURE 2: The expression levels of NCF1, NCF2, and NCF4 in subgroups of patients with KIRC. Relative expression of NCF1, NCF2, and NCF4 in $(a-c)$ normal individuals and patients with KIRC at different grades, $(d-f)$ normal individuals and patients with KIRC at different stages, and (g-i) normal individuals and patients with KIRC at different subtypes. ${ }^{*} p<0.05,{ }^{* *} p<0.01$, and ${ }^{* * *} p<0.001$.

Figures $1(\mathrm{~b})-1(\mathrm{~d}))$. Analysis of TCGA-KIRC samples in the UALCAN database revealed that mRNA expression levels of NCF1, NCF2, and NCF4 were significantly higher in KIRC tissues than in control tissues $(p<0.001$; Figures $1(\mathrm{e})-1(\mathrm{~g}))$.

3.2. The Levels of NCF1, NCF2, and NCF4 Expression in Subgroups of Patients with KIRC. To study various clinicopathological characteristics, we further analyzed TCGAKIRC samples in the UALCAN database. We next explored the different expression levels of the 3 genes in KIRC, stratified according to the cancer stage and pathological grade. The results demonstrated that the expression of the 3 genes was higher in KIRC tissues than in normal tissues based on different pathological grades and individual cancer stages. Therefore, expression levels of NCF1, NCF2, and NCF4 may serve as potential diagnostic markers in patients with KIRC (Figures 2(a)-2(f)). In addition, we also analyzed the mRNA expression levels of the NCFs in different subgroups of primary KIRC patients and healthy people. NCF1, NCF2, and NCF4 are significantly higher in $\mathrm{ccA}$ and $\mathrm{ccB}$ subtypes than normal tissues, and the expression of NCF1 and NCF4 in ccB subtypes is significantly higher than that in ccA subtypes (Figures 2(g)-2(i)). Therefore, the results indicated that the expression of these genes plays an important role in the tumorigenesis and progression of HCC.

3.3. Clinicopathological Characteristics and Prognostic Significance of NCF1, NCF2, and NCF4 Expression in KIRC. The transcriptome RNA-seq data from TCGA databases for 530 KIRC patients was collected. The clinicopathological characteristics of these kidney cancer patients are depicted in Table 1. Next, we sought to investigate the prognostic significance of NCF1, NCF2, and NCF4 expression in KIRC. The association between NCF1, NCF2, and NCF4 expression levels and the survival outcomes of KIRC patients was assessed using Kaplan-Meier survival curves. The results showed that patients were divided into two groups based on the expression values of NCF1, NCF2, and NCF4 in each 


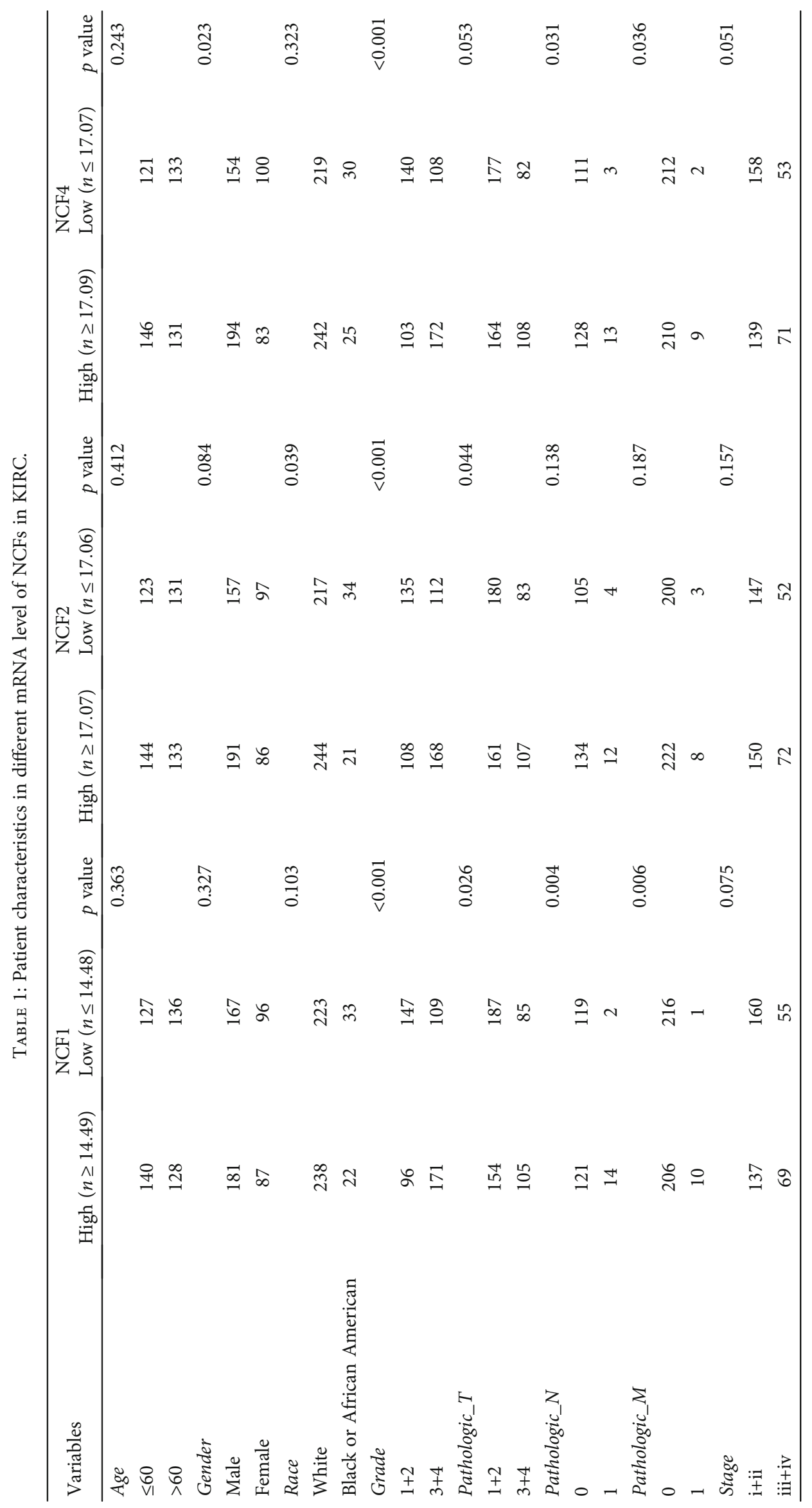



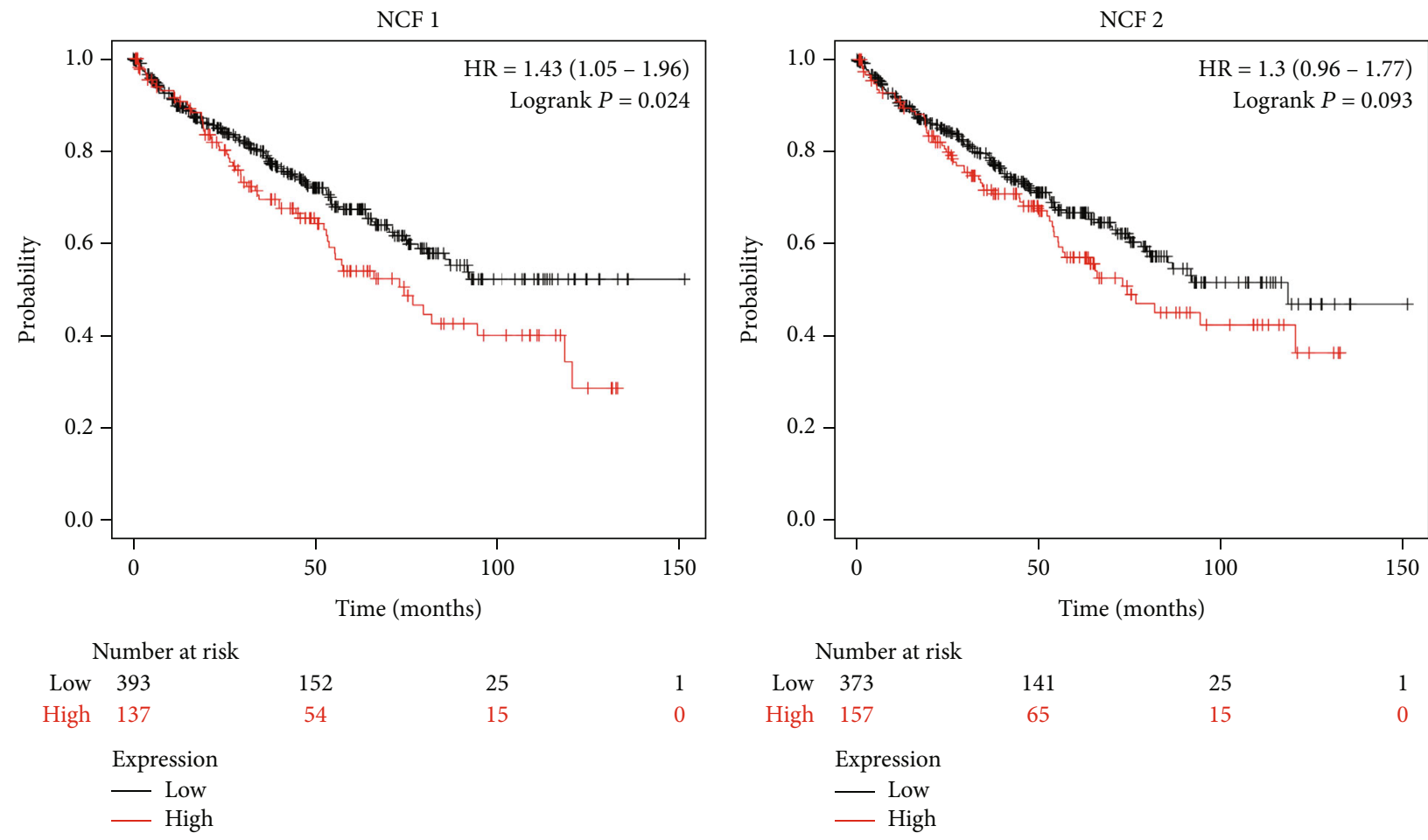

(a)

(b)

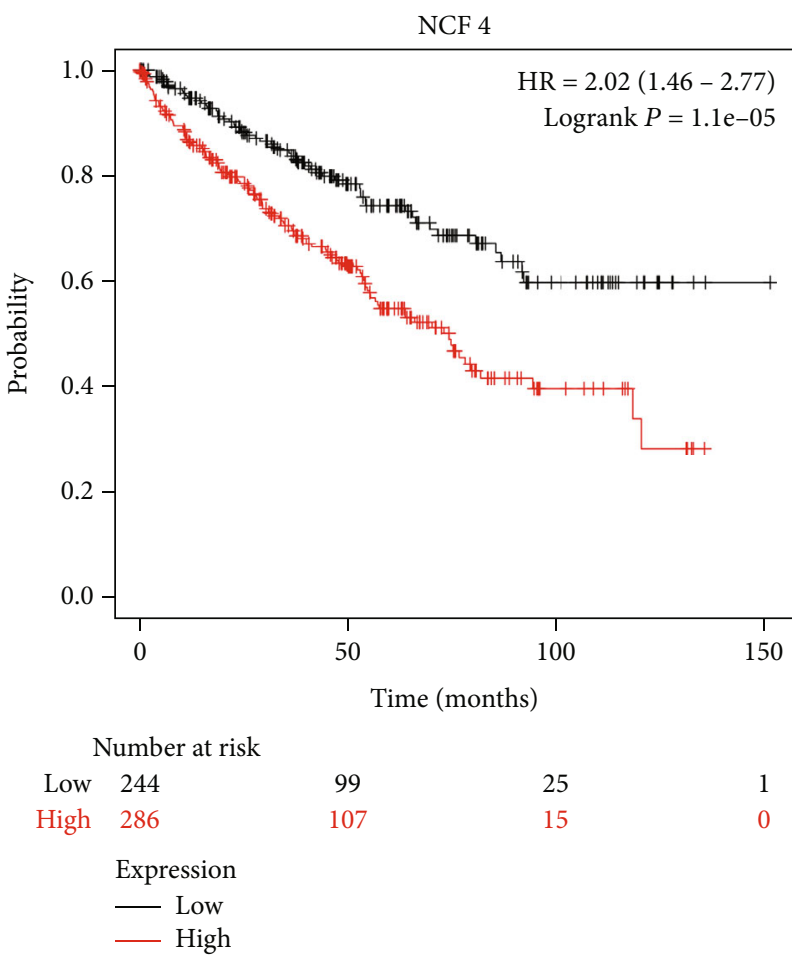

(c)

FIGURE 3: NCF1, NCF2, and NCF4 are associated with the overall survival (OS) rate in KIRC. (a-c) Kaplan-Meier survival curves comparing high- and low-expression levels of NCF1, NCF2, and NCF4 in KIRC using Kaplan-Meier Plotter database. Survival curves based on overall survival (OS).

cohort (autoselect the best cutoff). The group with high NCF1 and NCF4 expression had significantly shorter overall survival (OS) compared to the group with low expression of
NCF1, NCF2, and NCF4 based on TCGA data using the GEPIA database (Figure 3). These results strongly highlight the prognostic value of NCF1, NCF2, and NCF4 in KIRC. 


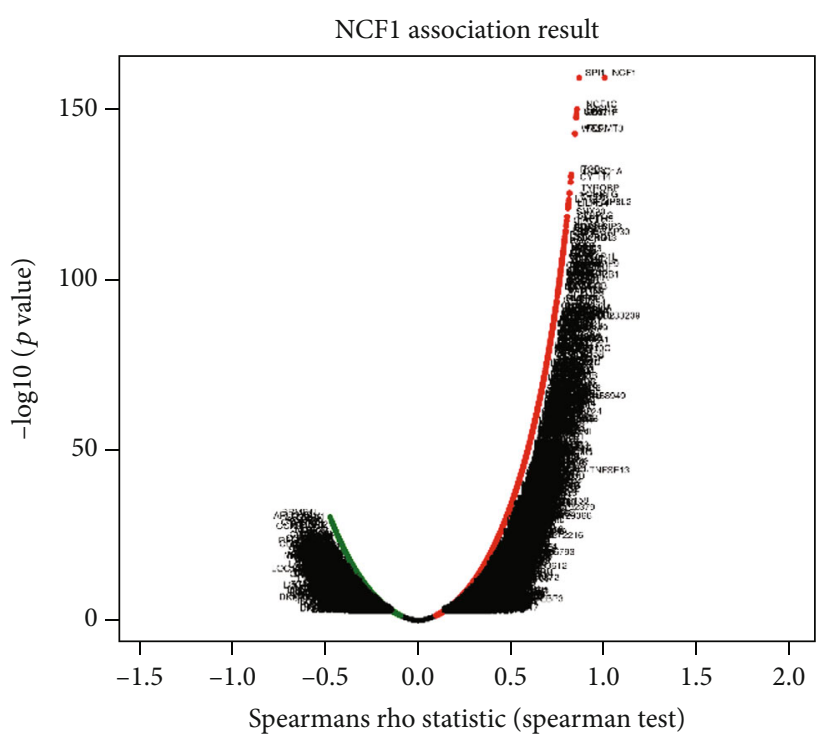

(a)

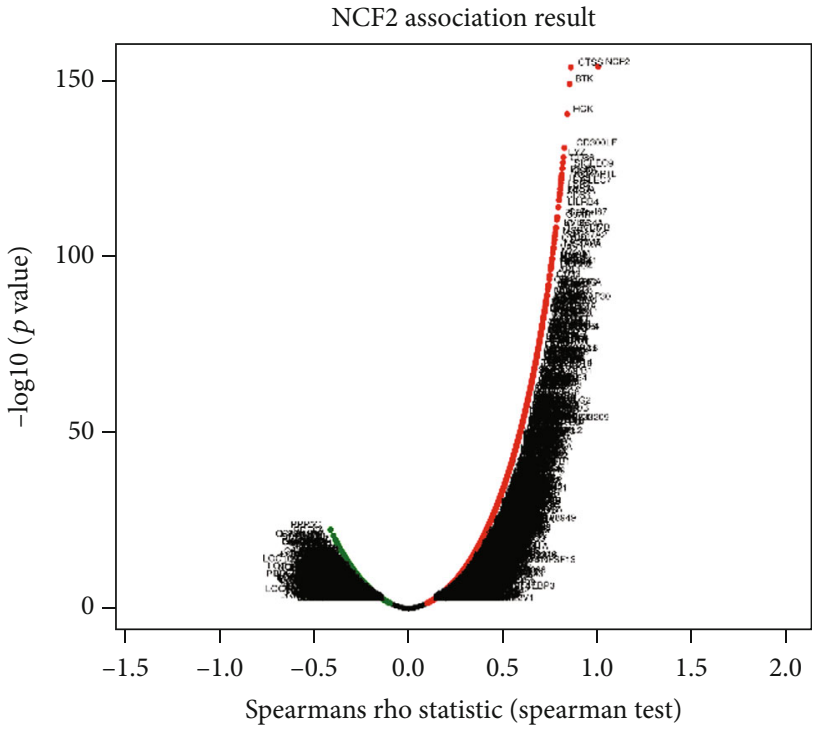

(b)

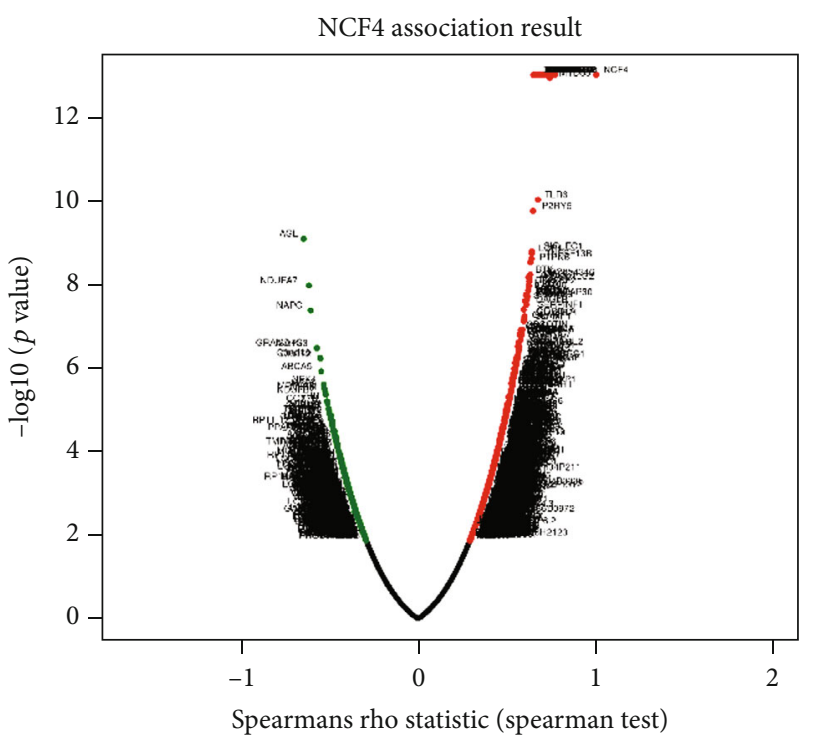

(c)

FIgUre 4: Continued. 


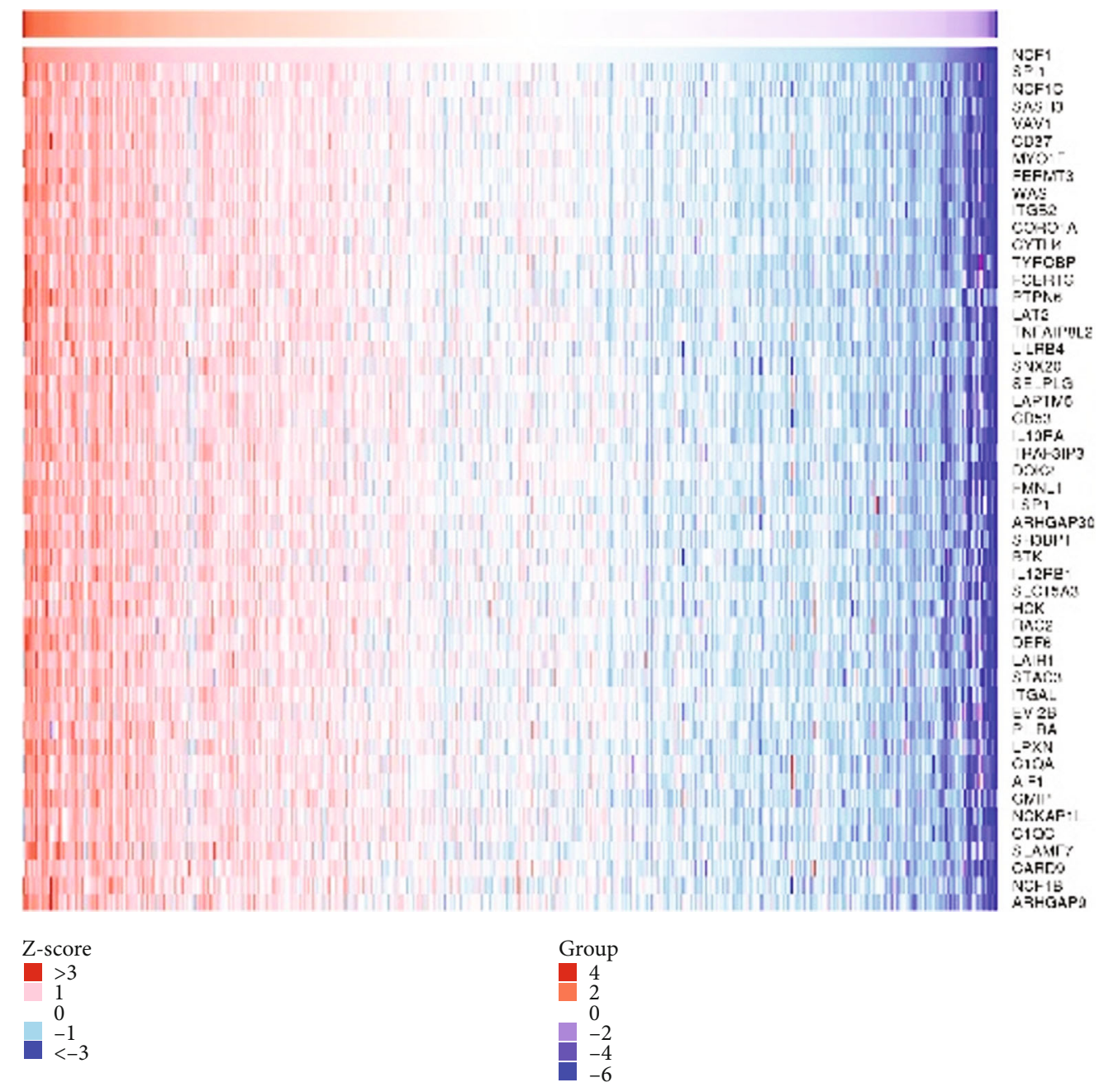

(d)

FIgUre 4: Continued. 


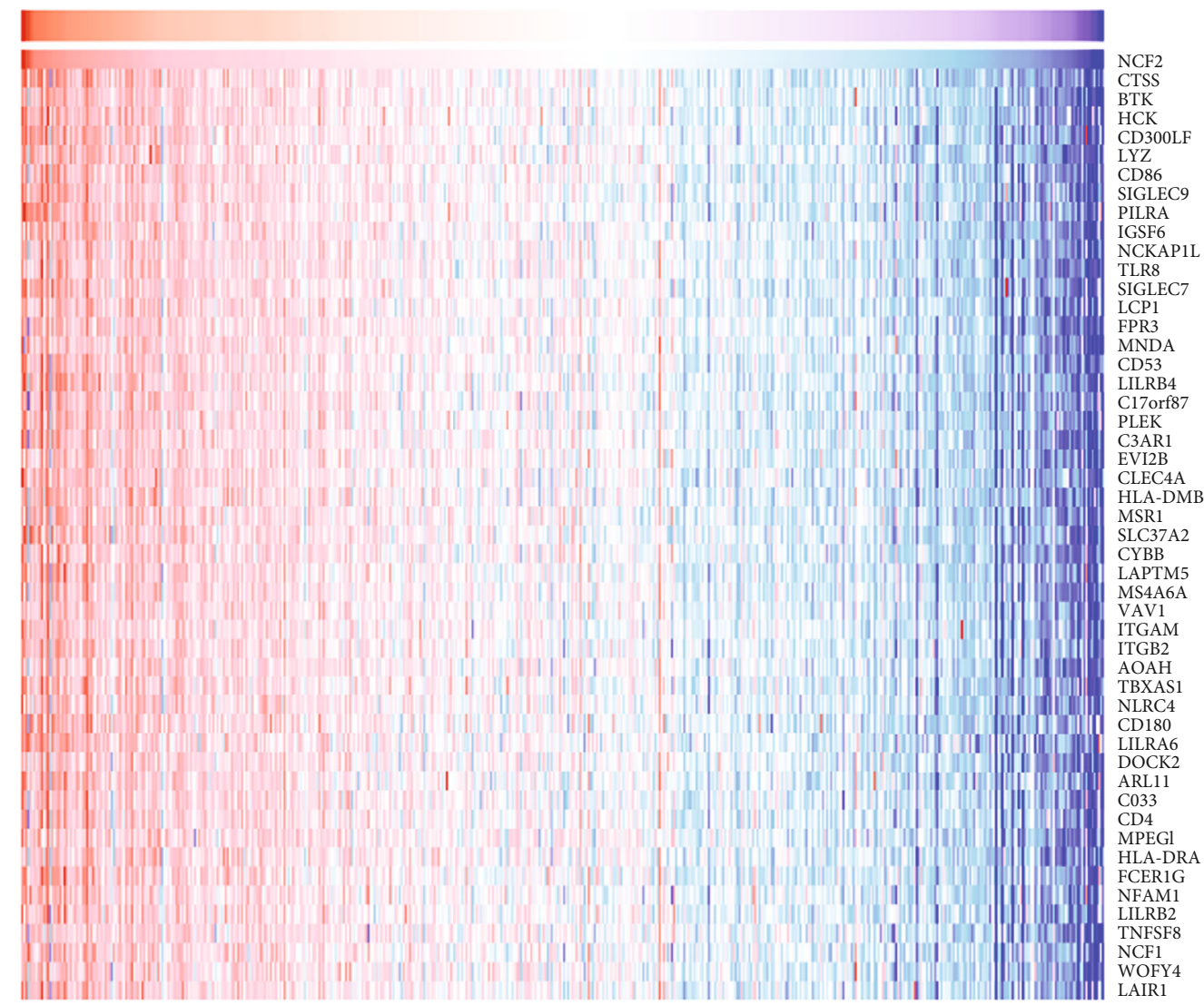

Z-score
$>3$
1
0
-1
$<-3$

Group

4
2

0
-2
-4

(e)

Figure 4: Continued. 


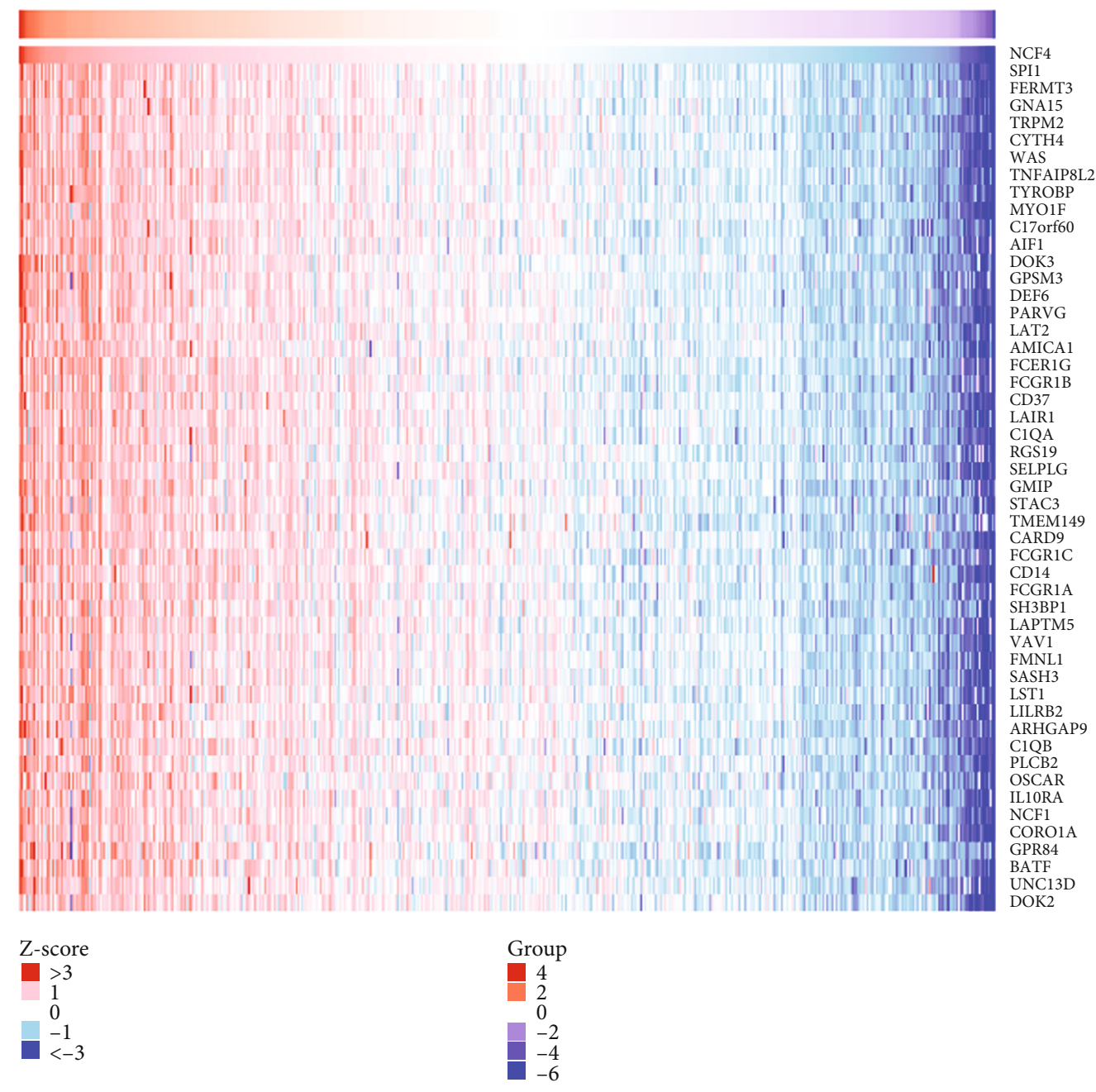

(f)

FIgUre 4: Continued. 


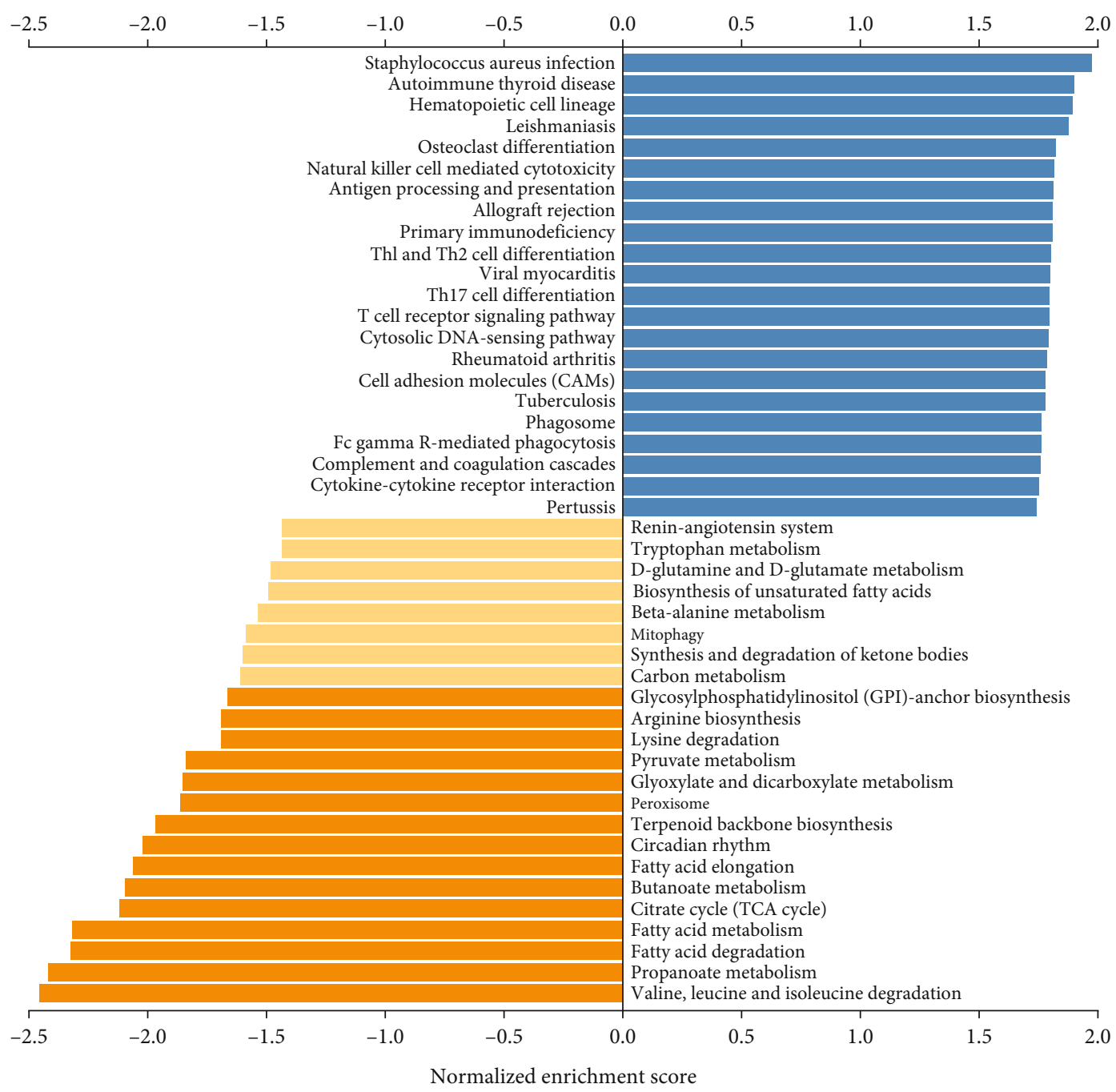

(g)

Figure 4: Continued. 


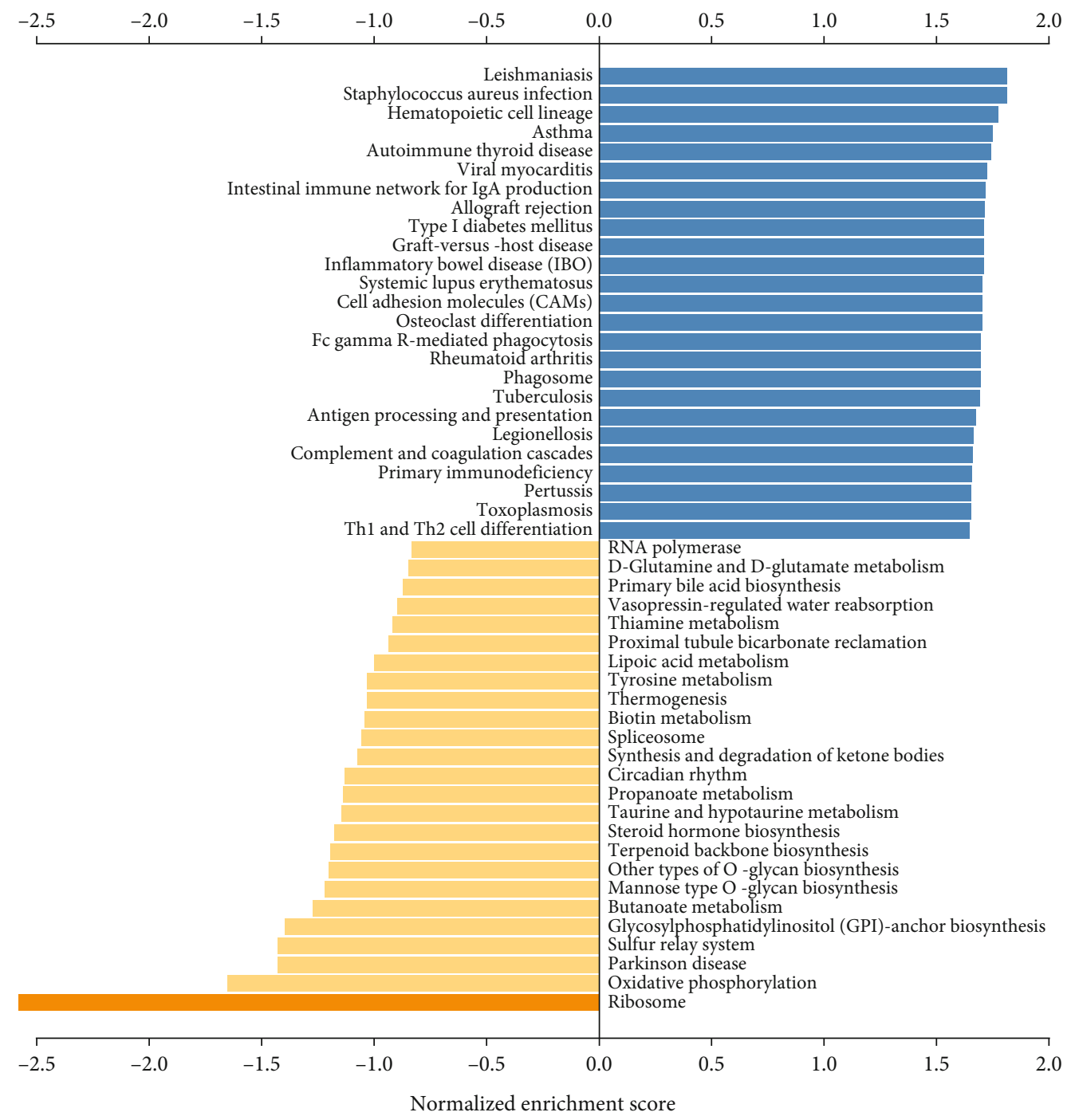

(h)

Figure 4: Continued. 


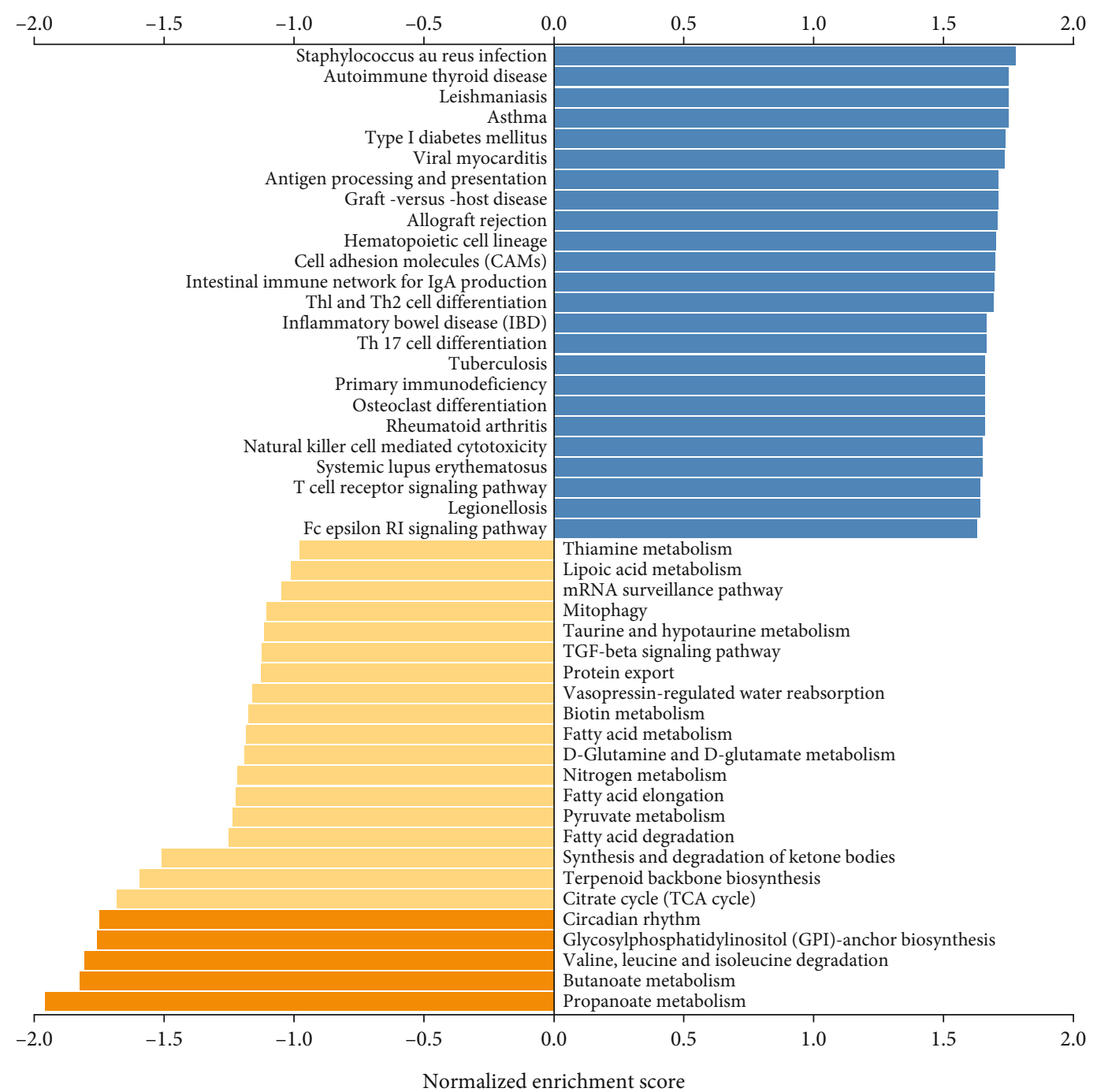

(i)

FIGURE 4: Differentially expressed genes and gene set enrichment analysis (GSEA) with NCF1, NCF2, and NCF4 high and low expression groups. (a-c) The NCF1, NCF2, and NCF4 highly correlated genes identified by Pearson test in KIRC cohort. (d-f) Heat map showing positive and negative correlations of the first 50 genes with NCF1, NCF2, and NCF4 in KIRC. Red indicates positively related genes, and blue indicates negatively related genes. (g-i) Significantly enriched KEGG pathways of NCF1, NCF2, and NCF4 in KIRC.

3.4. Alterations of NCF1, NCF2, and NCF4 Expression Networks in KIRC. Since gene regulation networks reflect common genetic risk factors that compose functional relationships, we investigated the regulatory factors related to NCF1, NCF2, and NCF4 in KIRC. Figures 4(a)-4(c) show genes highly coexpressed with NCF1, NCF2, and NCF4 based on Pearson correlation; genes positively and negatively correlated with NCF1, NCF2, and NCF4 are marked in the dark red and dark blue dots, respectively (FDR $<0.01$ ). The top 50 genes showing significant positive and negative correlation with NCF1, NCF2, and NCF4 are shown in heat maps (Figures 4(d)-4(f)).

In addition, results from the LinkedOmics database showed that the KEGG pathway related to NCFs mainly focused on immune activation and immune regulation function (Figures 4(g)-4(i)).

High expression of NCF1 was associated with adaptive immune response (BP category), immunological synapse (CC category), MHC protein binding (MF category), and Staphylococcus aureus infection (KEGG category). High expression of NCF2 was associated with detection of biotic stimulus (BP category), MHC protein complex (CC category), pattern recognition receptor activity (MF category), and Staphylococcus aureus infection (KEGG category). High expression of NCF4 was associated with adaptive immune response (BP category), immunological synapse (CC category), immunoglobulin binding ( $\mathrm{MF}$ category), and 
TABLE 2: Enrichment of NCF1, NCF2, and NCF4 in KIRC (DAVID). The diagram showed the GO enrichment in cellular component (CC) terms, biological process (BP) terms, and molecular function (MF) terms and bar plot of Kyoto Encyclopedia of Genes and Genomes (KEGG) enriched terms.

\begin{tabular}{|c|c|c|c|c|c|c|c|}
\hline Gene & Category & Gene set & Description & Size & ES & $p$ value & FDR \\
\hline \multirow{20}{*}{ NCF1 } & \multirow{5}{*}{$\mathrm{BP}$} & GO:0002250 & Adaptive immune response & 121 & 0.75491 & $<0.001$ & $<0.001$ \\
\hline & & GO:0002449 & Lymphocyte-mediated immunity & 85 & 0.73747 & $<0.001$ & $<0.001$ \\
\hline & & GO:0002694 & Regulation of leukocyte activation & 167 & 0.70281 & $<0.001$ & $<0.001$ \\
\hline & & GO:0007159 & Leukocyte cell-cell adhesion & 112 & 0.72889 & $<0.001$ & $<0.001$ \\
\hline & & GO:0002764 & Immune response-regulating signaling pathway & 159 & 0.70894 & $<0.001$ & $<0.001$ \\
\hline & \multirow{6}{*}{$\mathrm{CC}$} & GO:0001772 & Immunological synapse & 32 & 0.89059 & $<0.001$ & $<0.001$ \\
\hline & & GO:0042611 & MHC protein complex & 19 & 0.92416 & $<0.001$ & 0.0016732 \\
\hline & & GO:0098636 & Protein complex involved in cell adhesion & 35 & 0.83931 & $<0.001$ & 0.0037646 \\
\hline & & GO:0070820 & Tertiary granule & 155 & 0.8063 & $<0.001$ & 0.0046849 \\
\hline & & GO:0001891 & Phagocytic cup & 21 & 0.8995 & $<0.001$ & 0.0050195 \\
\hline & & GO:0042287 & MHC protein binding & 24 & 0.923 & $<0.001$ & $<0.001$ \\
\hline & \multirow{4}{*}{ MF } & GO:0003823 & Antigen binding & 52 & 0.84395 & $<0.001$ & 0.0033726 \\
\hline & & GO:0019865 & Immunoglobulin binding & 22 & 0.91359 & $<0.001$ & 0.0036792 \\
\hline & & GO:0035586 & Purinergic receptor activity & 26 & 0.86795 & 0.0023095 & 0.0040471 \\
\hline & & GO:0052813 & Phosphatidylinositol bisphosphate kinase activity & 74 & 0.82566 & $<0.001$ & 0.0044676 \\
\hline & \multirow{5}{*}{ KEGG } & hsa05150 & Staphylococcus aureus infection & 52 & 0.92179 & $<0.001$ & $<0.001$ \\
\hline & & hsa05416 & Viral myocarditis & 56 & 0.90004 & $<0.001$ & $<0.001$ \\
\hline & & hsa05320 & Autoimmune thyroid disease & 50 & 0.90708 & $<0.001$ & $<0.001$ \\
\hline & & hsa05140 & Leishmaniasis & 71 & 0.89 & $<0.001$ & $<0.001$ \\
\hline & & hsa04940 & Type I diabetes mellitus & 41 & 0.90434 & $<0.001$ & $<0.001$ \\
\hline \multirow{19}{*}{ NCF2 } & \multirow{5}{*}{$\mathrm{BP}$} & GO:0009595 & Detection of biotic stimulus & 21 & 0.95106 & $<0.001$ & $<0.001$ \\
\hline & & GO:0033108 & Mitochondrial respiratory chain complex assembly & 68 & -0.66273 & $<0.001$ & $<0.001$ \\
\hline & & GO:0010257 & NADH dehydrogenase complex assembly & 49 & -0.73433 & $<0.001$ & $<0.001$ \\
\hline & & GO:0042116 & Macrophage activation & 78 & 0.8476 & $<0.001$ & 0.00047859 \\
\hline & & GO:0042107 & Cytokine metabolic process & 106 & 0.848 & $<0.001$ & 0.0004994 \\
\hline & \multirow{6}{*}{$\mathrm{CC}$} & GO:0042611 & MHC protein complex & 19 & 0.93646 & $<0.001$ & $<0.001$ \\
\hline & & GO:0030964 & NADH dehydrogenase complex & 43 & -0.65251 & $<0.001$ & $<0.001$ \\
\hline & & GO:0098798 & Mitochondrial protein complex & 213 & -0.5402 & $<0.001$ & $<0.001$ \\
\hline & & GO:0001772 & Immunological synapse & 32 & 0.87477 & $<0.001$ & 0.00058165 \\
\hline & & GO:0070820 & Tertiary granule & 155 & 0.84456 & $<0.001$ & 0.00087247 \\
\hline & & GO:0038187 & Pattern recognition receptor activity & 20 & 0.94123 & $<0.001$ & $<0.001$ \\
\hline & \multirow{4}{*}{ MF } & GO:0042287 & MHC protein binding & 24 & 0.91201 & $<0.001$ & 0.00094517 \\
\hline & & GO:0003823 & Antigen binding & 52 & 0.87062 & $<0.001$ & 0.00094517 \\
\hline & & GO:0019865 & Immunoglobulin binding & 22 & 0.93547 & $<0.001$ & 0.0012602 \\
\hline & & GO:0043394 & Proteoglycan binding & 33 & 0.87104 & $<0.001$ & 0.0040957 \\
\hline & \multirow{5}{*}{ KEGG } & hsa05150 & Staphylococcus aureus infection & 52 & 0.92654 & $<0.001$ & $<0.001$ \\
\hline & & hsa05140 & Leishmaniasis & 71 & 0.90715 & $<0.001$ & $<0.001$ \\
\hline & & hsa05310 & Asthma & 28 & 0.93861 & $<0.001$ & $<0.001$ \\
\hline & & hsa05322 & Systemic lupus erythematosus & 122 & 0.86618 & $<0.001$ & $<0.001$ \\
\hline \multirow{8}{*}{ NCF4 } & & hsa04640 & Hematopoietic cell lineage & 93 & 0.89299 & $<0.001$ & $<0.001$ \\
\hline & \multirow{5}{*}{$\mathrm{BP}$} & GO:0002250 & Adaptive immune response & 368 & 0.78931 & $<0.001$ & $<0.001$ \\
\hline & & GO:0032609 & Interferon-gamma production & 102 & 0.79916 & $<0.001$ & $<0.001$ \\
\hline & & GO:0032613 & Interleukin-10 production & 46 & 0.82482 & $<0.001$ & $<0.001$ \\
\hline & & GO:0042110 & $\mathrm{T}$ cell activation & 439 & 0.76892 & $<0.001$ & $<0.001$ \\
\hline & & GO:0007159 & Leukocyte cell-cell adhesion & 310 & 0.76451 & $<0.001$ & $<0.001$ \\
\hline & \multirow{2}{*}{$\mathrm{CC}$} & GO:0001772 & Immunological synapse & 32 & 0.83629 & $<0.001$ & $<0.001$ \\
\hline & & GO:0042579 & Microbody & 125 & -0.56583 & $<0.001$ & $<0.001$ \\
\hline
\end{tabular}


TABLE 2: Continued.

\begin{tabular}{|c|c|c|c|c|c|c|c|}
\hline Gene & Category & Gene set & Description & Size & ES & $p$ value & FDR \\
\hline & \multirow{8}{*}{ MF } & GO:0001891 & Phagocytic cup & 21 & 0.85403 & $<0.001$ & 0.00053463 \\
\hline & & GO:0070820 & Tertiary granule & 155 & 0.72836 & $<0.001$ & 0.00080194 \\
\hline & & GO:0042611 & MHC protein complex & 19 & 0.84613 & $<0.001$ & 0.0016039 \\
\hline & & GO:0019865 & Immunoglobulin binding & 22 & 0.88392 & $<0.001$ & $<0.001$ \\
\hline & & GO:0016903 & $\begin{array}{l}\text { Oxidoreductase activity, acting on the aldehyde } \\
\text { or oxo group of donors }\end{array}$ & 43 & -0.62542 & $<0.001$ & $<0.001$ \\
\hline & & GO:0042287 & MHC protein binding & 24 & 0.85887 & $<0.001$ & 0.0011573 \\
\hline & & GO:0004896 & Cytokine receptor activity & 88 & 0.75566 & $<0.001$ & 0.001736 \\
\hline & & GO:0019955 & Cytokine binding & 119 & 0.69907 & $<0.001$ & 0.009982 \\
\hline & \multirow{5}{*}{ KEGG } & hsa05150 & Staphylococcus aureus infection & 52 & 0.88097 & $<0.001$ & $<0.001$ \\
\hline & & hsa04640 & Hematopoietic cell lineage & 93 & 0.80875 & $<0.001$ & $<0.001$ \\
\hline & & hsa05320 & Autoimmune thyroid disease & 50 & 0.84733 & $<0.001$ & $<0.001$ \\
\hline & & hsa04672 & Intestinal immune network for IgA production & 45 & 0.83546 & $<0.001$ & $<0.001$ \\
\hline & & hsa05140 & Leishmaniasis & 71 & 0.80899 & $<0.001$ & $<0.001$ \\
\hline
\end{tabular}

Staphylococcus aureus infection (KEGG category). More enrichment pathways are shown in Table 2.

3.5. Genomic Alterations of NCF1, NCF2, and NCF4 in KIRC. The frequency and types of genetic alterations in NCF1, NCF2, and NCF4 in patients with HCC were analyzed by using the cBioPortal database. A total of $274 \mathrm{KIRC}$ cases from TCGA were explored. NCF1, NCF2, and NCF4 were altered in $4 \%, 7 \%$, and $5 \%$ of KIRC cases, respectively (Figure 5(a)). NCF1 mutation consisted of $4.01 \%$ mRNA high (11 cases). NCF2 mutation consisted of $0.36 \%$ mutation ( 1 case), $0.36 \%$ deep deletion (1 case), and 5.84\% mRNA high (16 cases). NCF4 mutation consisted of $0.36 \%$ mutation ( 1 case), $0.36 \%$ deep deletion (1 case), and $4.01 \%$ mRNA high (11 cases) (Figure 5(b)). NCF2 and NCF4 have existed mutations in the protein functional domain (Figure 5(c)). However, the copy number alteration status of NCF1, NCF2, and NCF4 was not significantly associated with the overall survival (OS) and disease-specific survival (DSS) of KIRC (Figures 5(d)-5(i)). In KIRC patients, the probability of mutations in NCF1, NCF2, and NCF4 is not high. We did not find that mutations may have beneficial or harmful effects on survival in this small sample of data. Therefore, genomic alterations of these genes could not be considered as poor prognosis factors in KIRC patients.

3.6. Correlation between NCF Expression and Immune Infiltrating Level in KIRC. The survival of patients in several cancers is determined by the number and activity of tumorinfiltrating lymphocytes. Therefore, the TIMER database was used to investigate the relationship between the levels of immune infiltrating and the expressions of NCF1, NCF2, and NCF4 in KIRC patients. As shown in Figure 6(a), high levels of NCF1, NCF2, and NCF4 mRNA expression had a significantly negative correlation with tumor purity (NCF1, $r=-0.284, p<0.001$; NCF2, $r=-$ $0.241, p<0.001$; and NCF4, $r=-0.357, p<0.001)$ in KIRC.
Overexpression of each of these genes was significantly associated with higher immune cell infiltration levels. Specifically, the NCF1, NCF2, and NCF4 expression level was positively correlated with infiltration levels of $\mathrm{CD}^{+} \mathrm{T}$ cells (NCF1, $r=0.406, p<0.001 ; \mathrm{NCF} 2, r=0.389, p<0.001$; and NCF4, $r=0.245, p<0.001), \mathrm{CD}^{+} \mathrm{T}$ cells (NCF1, $r=$ $0.336, p<0.001$; NCF2, $r=0.328, p<0.001$; and NCF4, $r$ $=0.464, p<0.001)$, B cells (NCF1, $r=0.452, p<0.001$; NCF2, $r=0.425, p<0.001$; and NCF4, $r=0.265, p<0.001)$, macrophages (NCF1, $r=0.466, p<0.001$; NCF2, $r=0.672, p$ $<0.001$; and NCF4, $r=0.559, p<0.001)$, neutrophils (NCF1, $r=0.566, p<0.001$; NCF2, $r=0.683, p<0.001$; and NCF4, $r=0.575, p<0.001$ ), and DCs (NCF1, $r=0.636, p$ $<0.001$; NCF2, $r=0.726, p<0.001$; and NCF4, $r=0.528$, $p<0.001$ ) (Figures 6(b)-6(d)). Specifically, NCF2 expression was not correlated with immune subtypes (C1-C6: wound healing, IFN-gamma dominant, inflammatory, lymphocyte depleted, and TGF- $\beta$ dominant) in KIRC. In addition, it was found that the mRNA levels of NCF1 and NCF4 were obviously decreased in immunologically quiet KIRC immune subtype.

Moreover, the relationships between somatic copy number alterations (SCNA) of the 3 genes and tumor infiltration levels among KIRC were investigated. Interestingly, the results showed that the CNA of NCF1 had significant correlations with the infiltration levels of $\mathrm{CD} 4^{+} \mathrm{T}$ cells and $\mathrm{B}$ cells; the CNA of NCF2 had significant correlations with $\mathrm{CD} 8^{+} \mathrm{T}$ cells, $\mathrm{CD}^{+}{ }^{+} \mathrm{T}$ cells, B cells, neutrophils, dendritic cells, and macrophages; and the CNA of NCF4 had a significant correlation with $\mathrm{CD}^{+} \mathrm{T}$ cells, $\mathrm{CD} 4^{+} \mathrm{T}$ cells, neutrophils, and dendritic cells (Figures 7(a)-7(c)).

3.7. Enrichment Analyses of NCFs in KIRC. We then performed enrichment analyses of NCFs. We collected NCF1, NCF2, and NCF4 and their coexpressed genes and plotted PPI plots with GeneMANIA (20) and STRING (10) (Figures 8(a) and 8(b)). The PPI network was constructed and revealed that NCFs were associated with osteoclast 
NCF1 $4 \%$ NCF2 7\% 7 . NCF4 5\% Genetic alteration

Missense mutation (unknown significance)

Truncating mutation (unknown significance)

II mRNA high

I Deep deletion

(a)

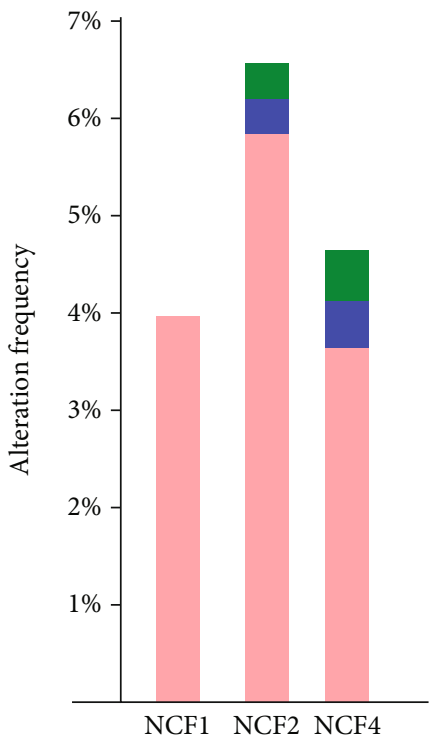

- Mutation

- Deep deletion

O mRNA high

+ Mutation data

+ CNA data

+ mRNA data

(b)
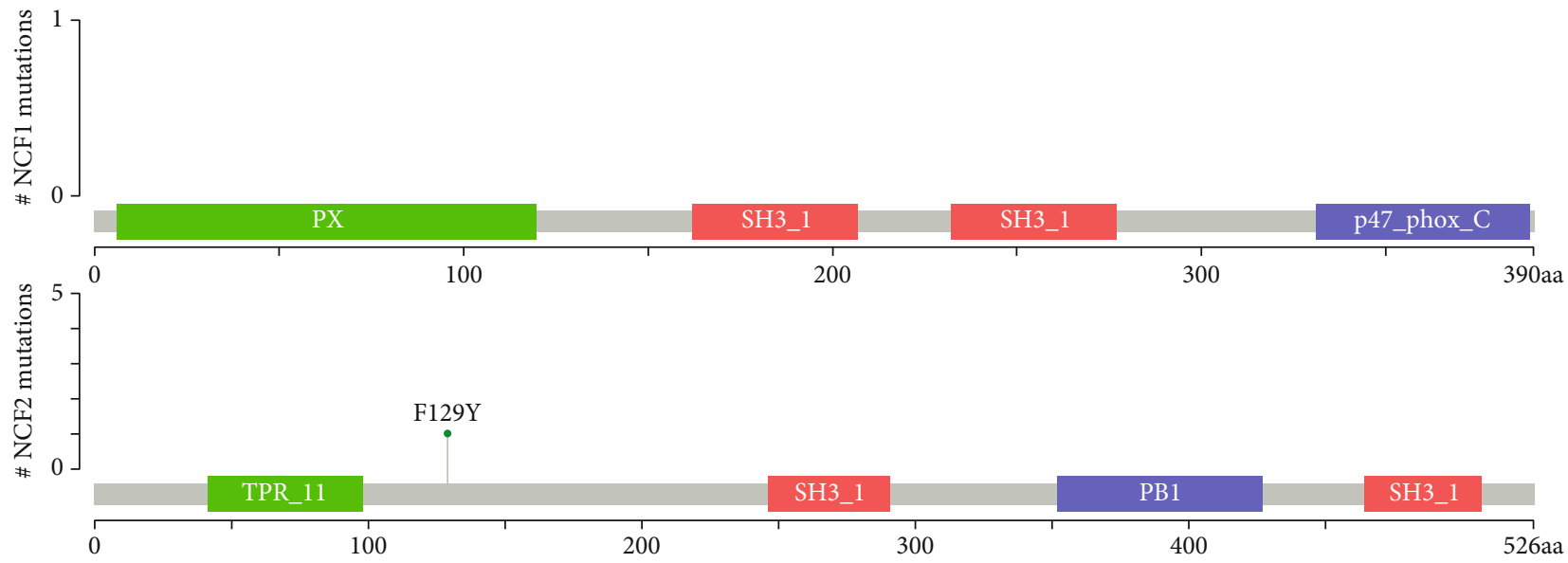

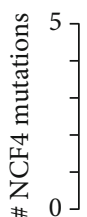

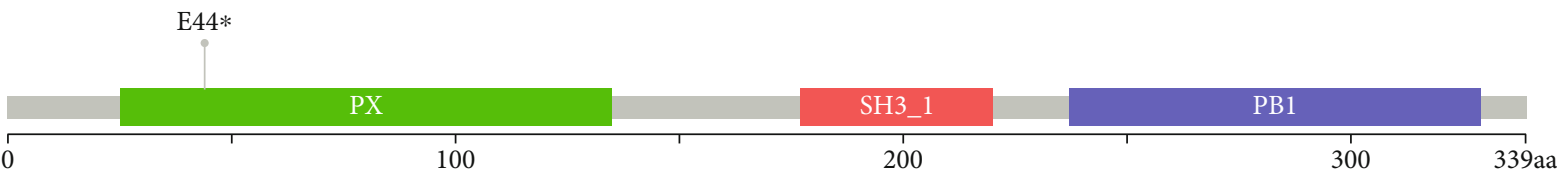

(c)

FIgUre 5: Continued. 


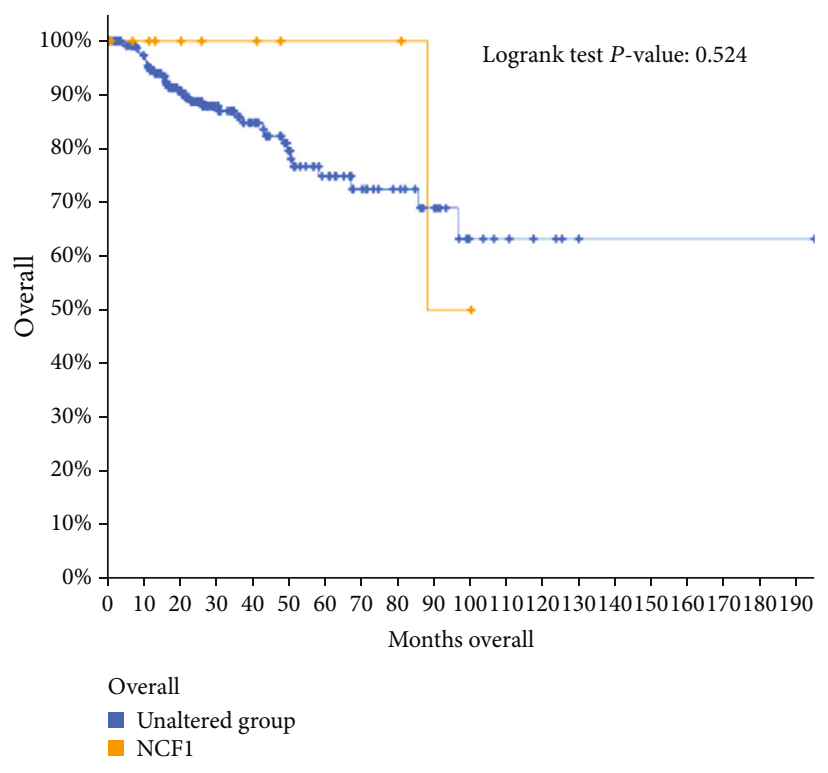

(d)

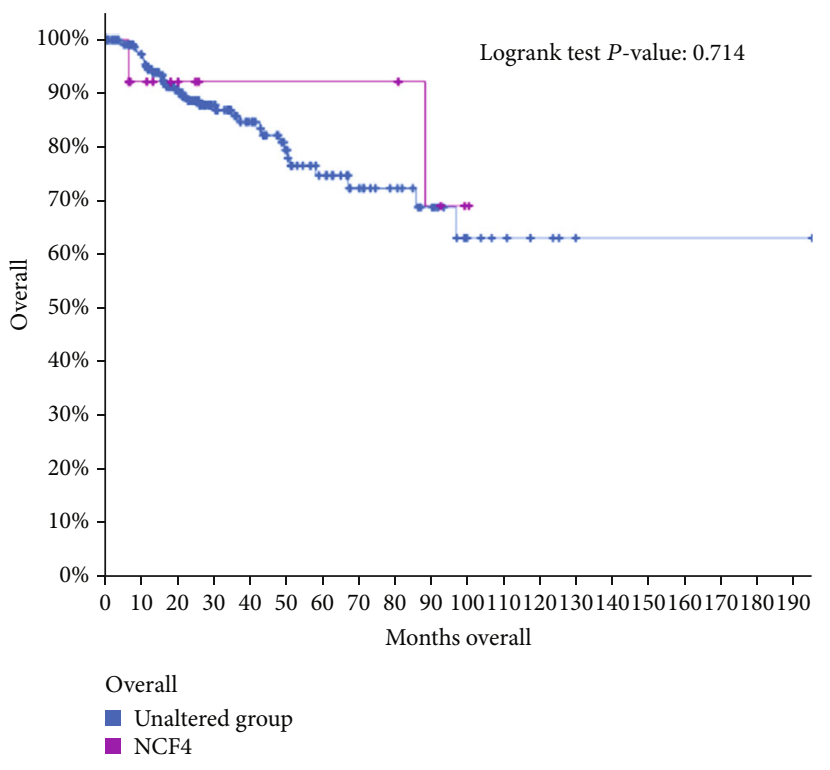

(f)

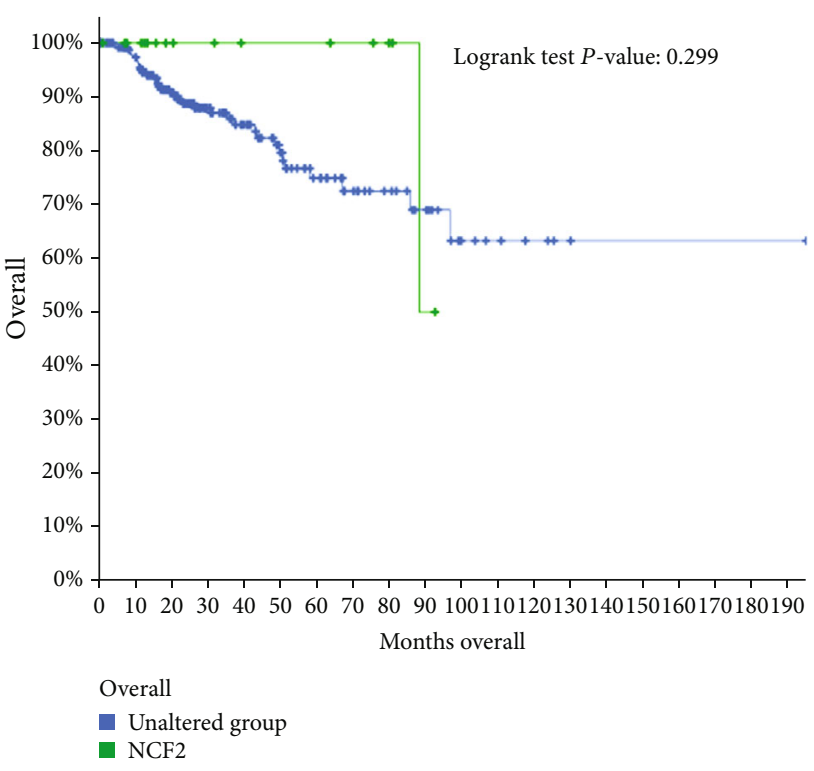

(e)

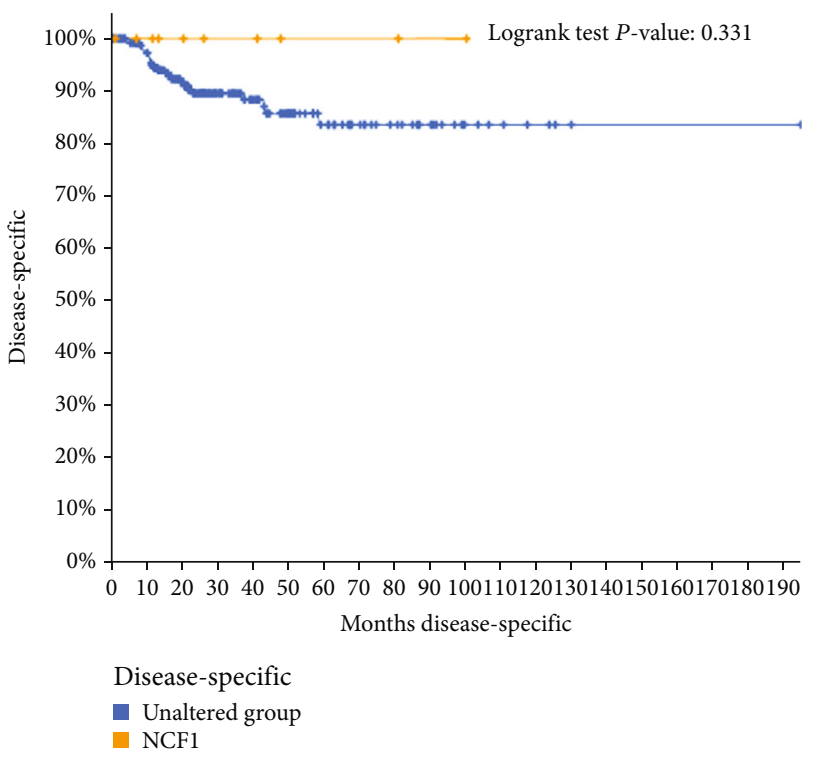

(g)

FIgURe 5: Continued. 


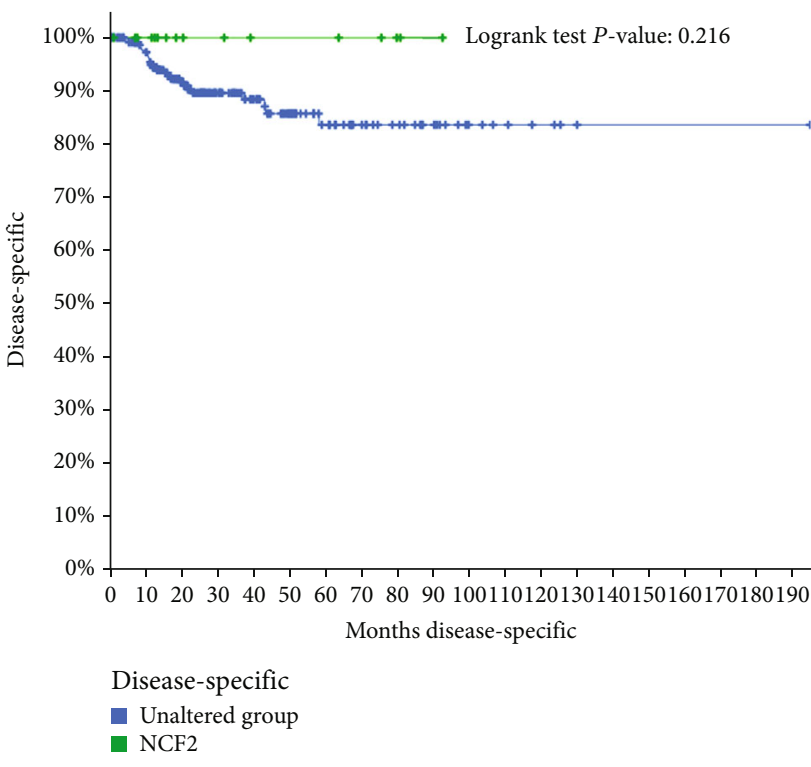

(h)

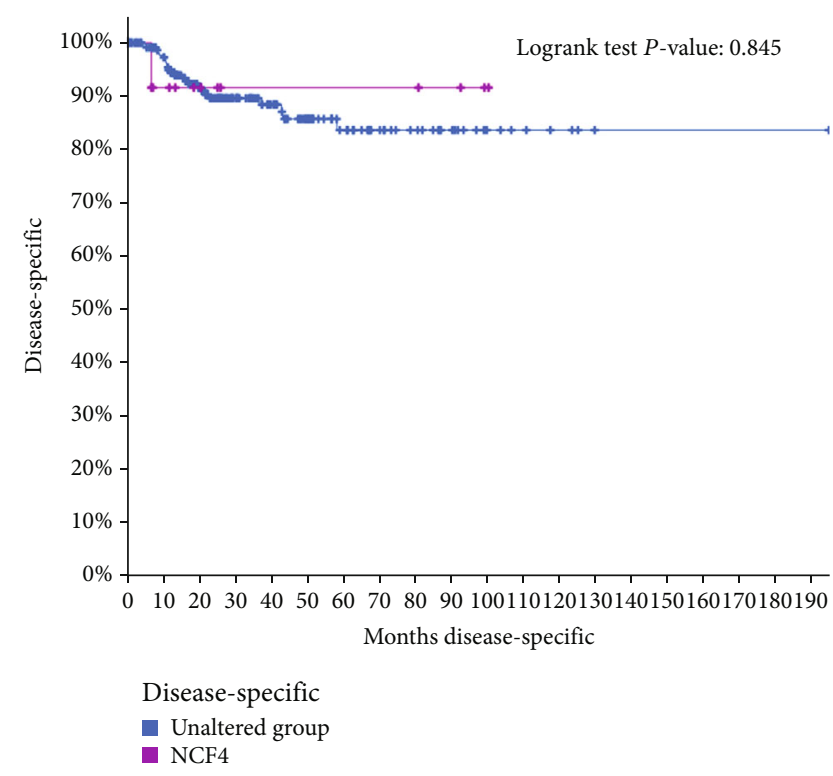

(i)

FIGURE 5: Genomic alterations of NCF1, NCF2, and NCF4 in KIRC. (a) OncoPrint of NCF1, NCF2, and NCF4 alterations in KIRC. Different types of genetic alterations highlighted in different colors, including mutation, mRNA high, and deep deletion. (b) Mutation frequency and copy number variations in the TCGA-BRCA dataset were determined using the cBioPortal website. (c) The mutation site legend of NCF1/2/4. (d-i) Association between cases with NCF1, NCF2, and NCF4 CNA status and overall survival (OS) and disease-specific survival (DSS) of patients with KIRC.

differentiation, natural killer cell-mediated cytotoxicity, MHC class I protein binding, GTPase activator activity, immunological synapse, and immune response.

To understand specifically the potential role of NCFs in the development of kidney renal clear cell carcinoma, we conducted GO and KEGG functional enrichment analysis of the top 100 correlated genes of distinct NCFs using DAVID which was extracted from the GEPIA (http://gepia .cancer-pku.cn/) database as presented in Table S1. We observed that all these three members were closely associated with immune-related biological functions, such as adaptive immune response, innate immune response, regulation of immune response, and integrin-mediated signaling pathway (Figure 8(c)). Therefore, we have reason to believe that NCF members may be related to the immune response in the tumor microenvironment (Figure 8(c)). In addition, we have also observed a higher correlation between NCFs and immunosuppressive immune infiltrating cells (Table 3).

\subsection{NCF4 Inhibition Blocks Kidney Cancer Cell Proliferation} In Vitro. We tested the expression of NCFs in human normal renal tubular epithelial cell line $\mathrm{HK}-2$ and KIRC cell line 786-O and found that NCF1, NCF2, and NCF4 were significantly upregulated in the KIRC cell line (Figure 9(a)). To verify the effects of NCF4 on the biological function of KIRC cells in vitro, the siRNAs were synthesized for knocking down the expression of NCF4 in the 786-O cell line (Figures 9(b) and 9(c)). Overexpression of NCF4 led to activation of the NADPH oxidase 2 complex and ROS production (activation of NADPH oxidase subunit NCF4 induces ROS-mediated EMT signaling in kidney cancer cells). The generation, location, and local concentration of free radicals in tumor cells play an important role in the biological behavior of tumors [29]. In order to explore the relationship between them, we measured the level of ROS after knocking down NCF4 in the 786-O cell line. NCF4 knockdown decreases the level of ROS in kidney cancer cells (Figure 9(d)). A CCK-8 assay indicated that cell viability was inhibited by silencing NCF4 and enhanced by exogenous hydrogen peroxide in 786-O cells (Figure 9(e)). The data of the colony formation assay also showed that exogenous hydrogen peroxide increased the proliferation, whereas silencing NCF4 inhibited the proliferation in kidney cancer cells (Figure 9(f)).

3.9. NCF4 Inhibition Blocks the Migration and EpithelialMesenchymal Transition of Kidney Cancer Cells In Vitro. Wound healing assays and transwell assays were conducted simultaneously after NCF4 knockdown to figure out the potential role of NCF4 in the migration and invasion capacity of bladder cancer cells. The results showed that knockdown of NCF4 significantly inhibited the ability of migration of KIRC cells (Figures 10(a) and 10(b)). Epithelial-mesenchymal transition (EMT) has been reported to play an important role in carcinoma metastasis, and due to EMT-mediated cell morphology changes, tumor cells are more likely to metastasize to distant places [30]. After confirming that NCF4 mediated KIRC cell migration, we investigated the EMT-relevant markers by western blotting. As the result showed, the expression of the epithelial marker E-cadherin was enhanced, and the mesenchymal markers vimentin and matrix metalloproteinase MMP9 that are closely correlated with metastasis were attenuated when NCF4 was knocked down (Figure 10(c)). 


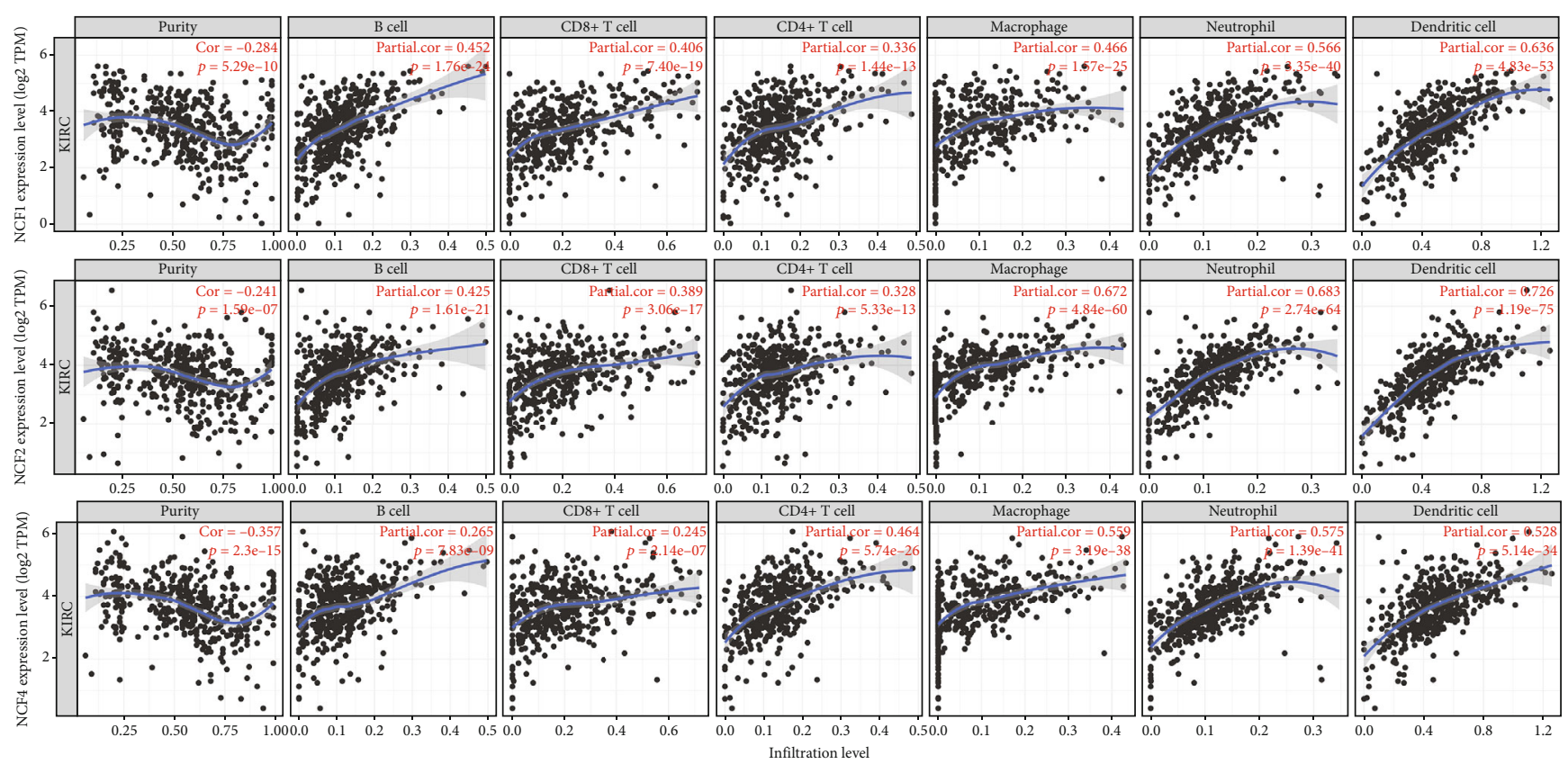

(a)

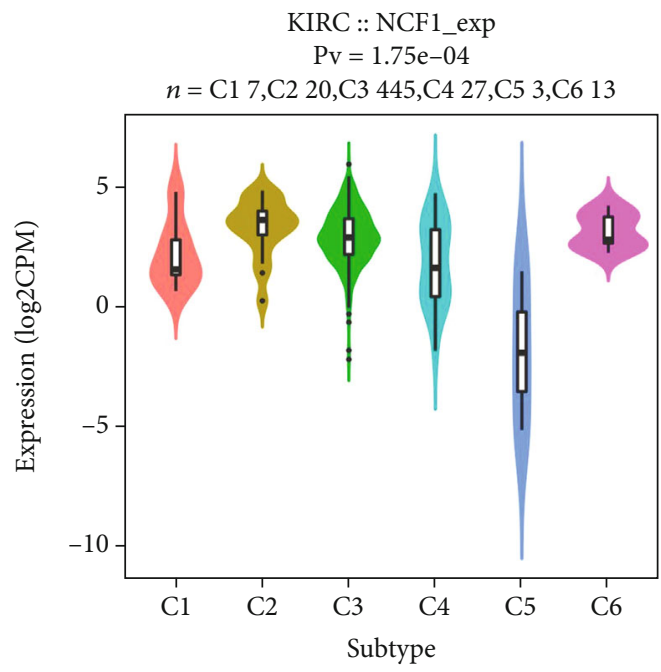

(b)
KIRC :: NCF2_exp

$\mathrm{Pv}=8.14 \mathrm{e}-02$

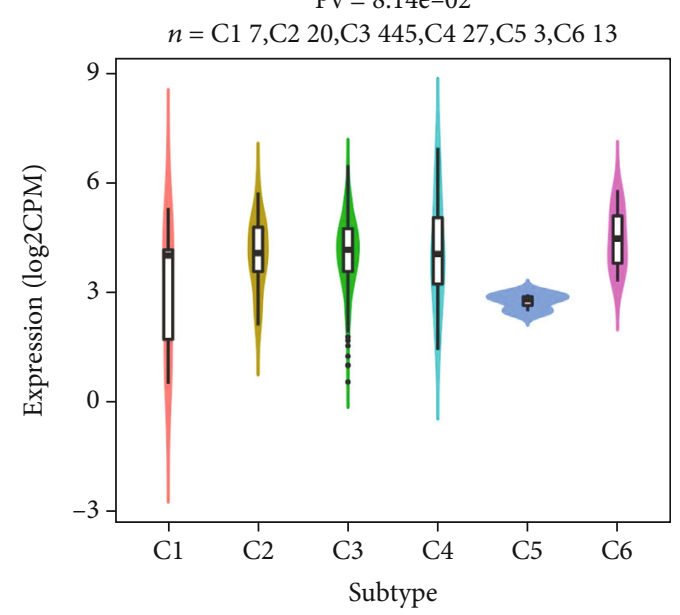

(c)

Figure 6: Continued. 


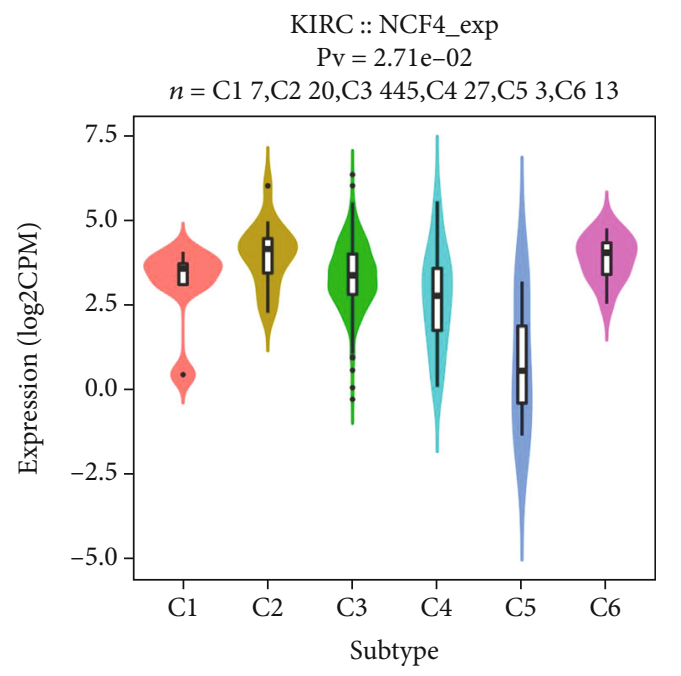

(d)

FIgURe 6: Correlation between NCF expression and immune infiltrating level in KIRC. (a) NCF1, NCF2, and NCF4 expression levels were significantly related to tumor purity and significant positive correlations existed with immune infiltration cells including CD ${ }^{+} \mathrm{T}$ cells, $\mathrm{CD} 8^{+}$ T cells, B cells, neutrophils, macrophages, and DCs in KIRC. (b-d) Correlation of NCF1, NCF2, and NCF4 expression and immune subtypes (C1-C6: wound healing, IFN-gamma dominant, inflammatory, lymphocyte depleted, and TGF- $\beta$ dominant) in KIRC.

\section{Discussion}

Clear cell RCC (KIRC) is a kind of silent cancer. The symptoms are not obvious in the early stage of the disease and it is difficult to be detected [31]. Therefore, the prognosis of this disease is often very bad [31]. RCC is showing an upward trend all over the world, especially in developed countries that maintain a high incidence [32]. At present, the research on early screening and molecular diagnosis of RCC is continuously deepening, and the diagnosis and treatment of RCC have been improved to a certain extent [33].

RCC is considered to be an immunogenic tumor, but it is known to mediate immune function to a large extent by causing immunosuppressive cells (such as regulatory $\mathrm{T}$ cells and suppressor cells of myeloid origin) to infiltrate the tumor microenvironment, considered as a "cold tumor" [34]. With the further in-depth research of TME, the receptors on the surface of immune cells and stromal cells in the tumor microenvironment have been recognized and studied more, which brings more opportunities and approaches for tumor treatment but also clarifies tumor progression. Therefore, more abnormally expressed genes with potential clinical relevance need to be considered for their possible effects, which will bring benefits to patients with more detailed diagnostic evaluation and treatment. In addition, the data of the past few decades clearly show that immunosuppressive changes in tumors are important tumor driving factors and immune escape mechanisms, which occur earlier. Tumor immunosuppression is usually found in cancer. Tumors mainly pass through two types of cells, regulatory $\mathrm{T}$ cells or Tregs and myeloid cells, called myeloid-derived suppressor cells (MDSC), which are immunosuppressive agents, which means that they secrete chemicals (such as cytokines) to inhibit infiltrating T cells [35].
In our study, NCF1, NCF2, and NCF4 showed a high expression in renal clear cell carcinoma. And we further proved that especially NCF1 and NCF4 were significantly correlated with high tumor grade and clinic stage. In addition, overexpression of NCF1, NCF2, and NCF4 was associated with shorter overall survival. So far, more and more evidence show that NCF1, NCF2, and NCF4 play an important role in tumorigenesis and development.

NOX2 is often referred to as NADPH oxidase, composed of the assembly of CYBB/gp91phox with the membraneanchored CYBA/p22phox and the cytosolic subunits NCF4/p40phox, NCF1/p47phox, and NCF2/p67phoxon the plasma membrane to generate extracellular ROS [16]. ROS affects the occurrence, development, and metastasis of cancer through a variety of mechanisms [16]. In recent years, a large number of studies have shown that altered ROS production may promote tumors, while other findings have also proved that ROS production can increase the sensitivity of cancer cells to various death-inducing pathways [36]. NCFs are the subunits of NOX2. Variations and deletions in any one subunit may induce changes in ROS production, which will affect tumors.

The study by van der Weyden et al. showed that NOX2 significantly affects the process of metastasis, because mice that are genetically deprived of any major NOX2 subunits (any subunit is necessary for NOX2 function) always after intravenous injection of tumor cells show a lower rate of lung metastasis [37]. Kelkka et al. found that NCF1 (m1J) mutant mice developed significantly smaller tumors in two melanoma models and tumor incidence was reduced in Lewis lung cancer tumors. The lack of ROS-mediated protection against tumor growth was associated with increasing immunityassociated cytokines [38]. NCF2 is significantly upregulated in RCC, as a potential factor predicting RCC [39]. 


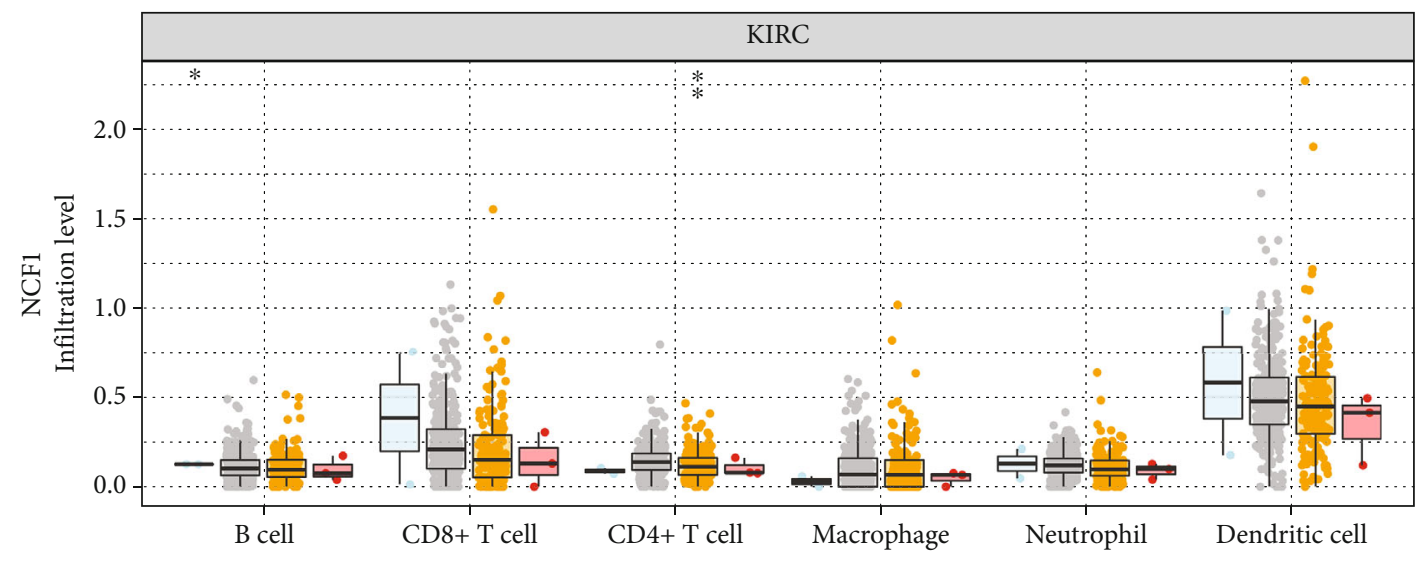

Copy number

Arm-level deletion

Diploid/normal

Arm-level gain

High amplication

(a)

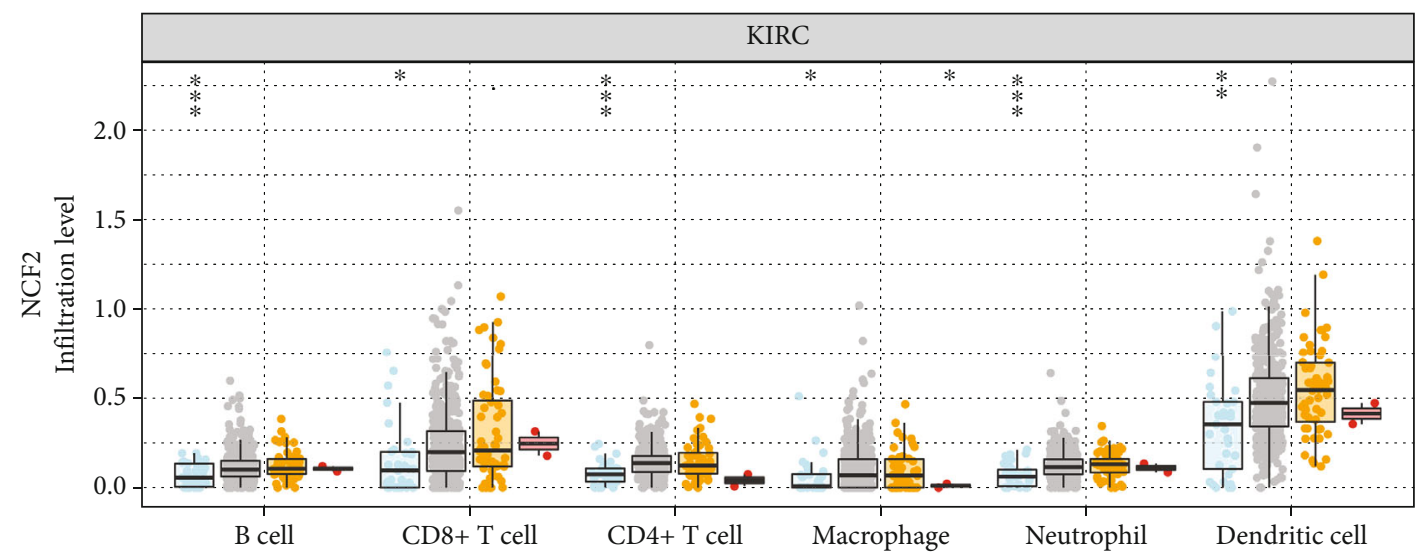

Copy number

Arm-level deletion

Arm-level gain

Diploid/normal

High amplication

(b)

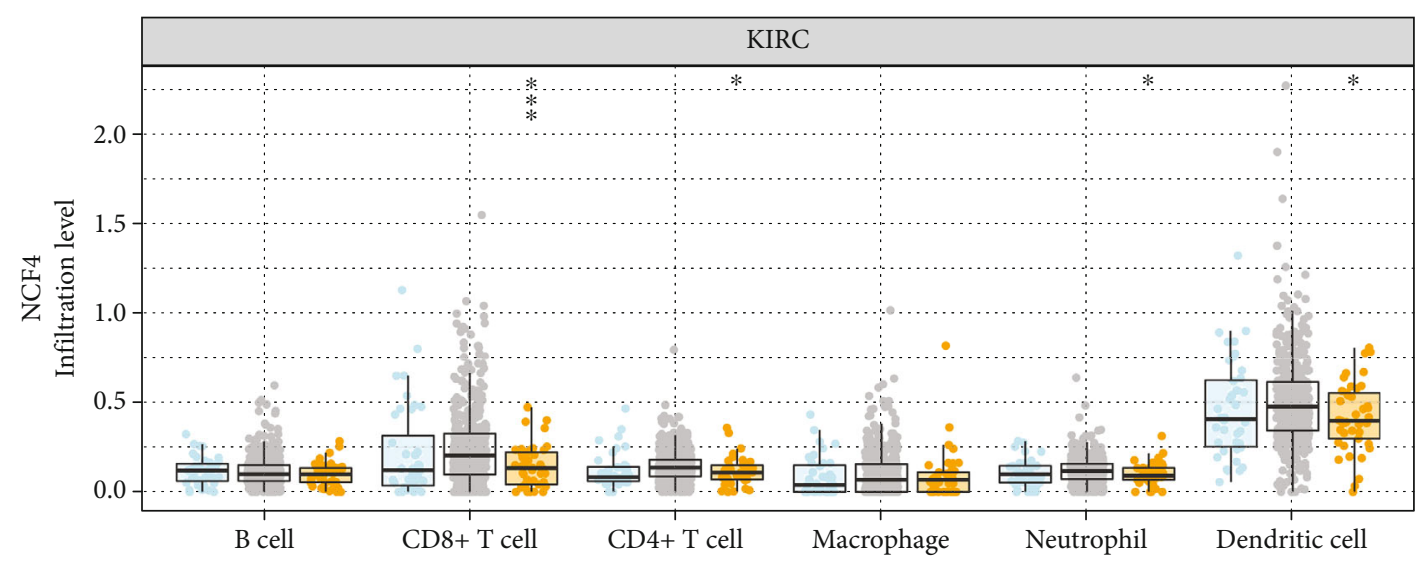

Copy number

Arm-level deletion

Diploid/normal

Arm-level gain

High amplication

(c)

FIGURE 7: The correlation between copy number alteration of NCFs and immune cell infiltration in KIRC. (a-c) CNA of NCF1, NCF2, and NCF4 had significant correlations with immune infiltration cells including CD4 ${ }^{+} \mathrm{T}$ cells and B cells. CNA: copy number alteration. 


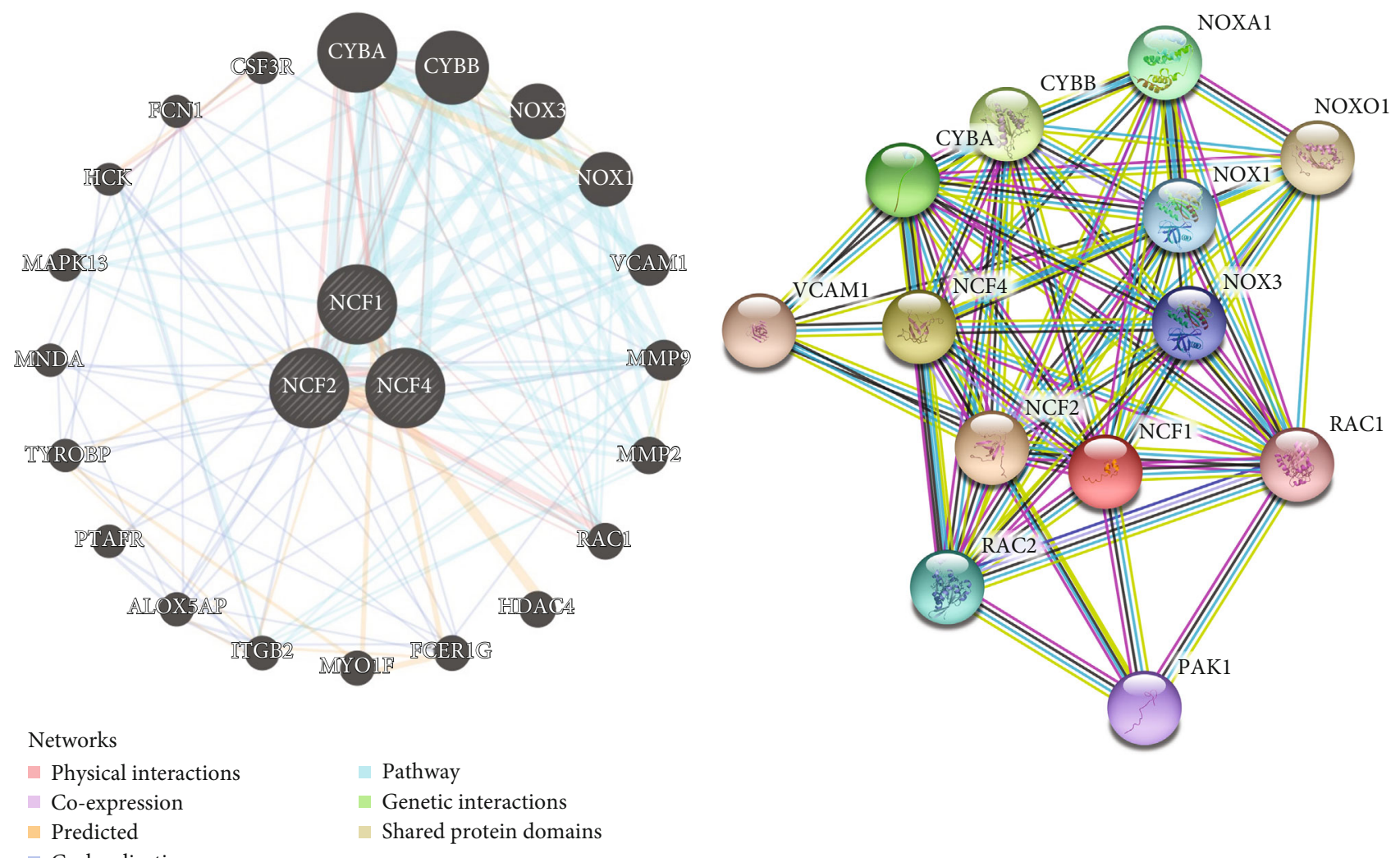

(a)

(b)

FIgure 8: Continued. 


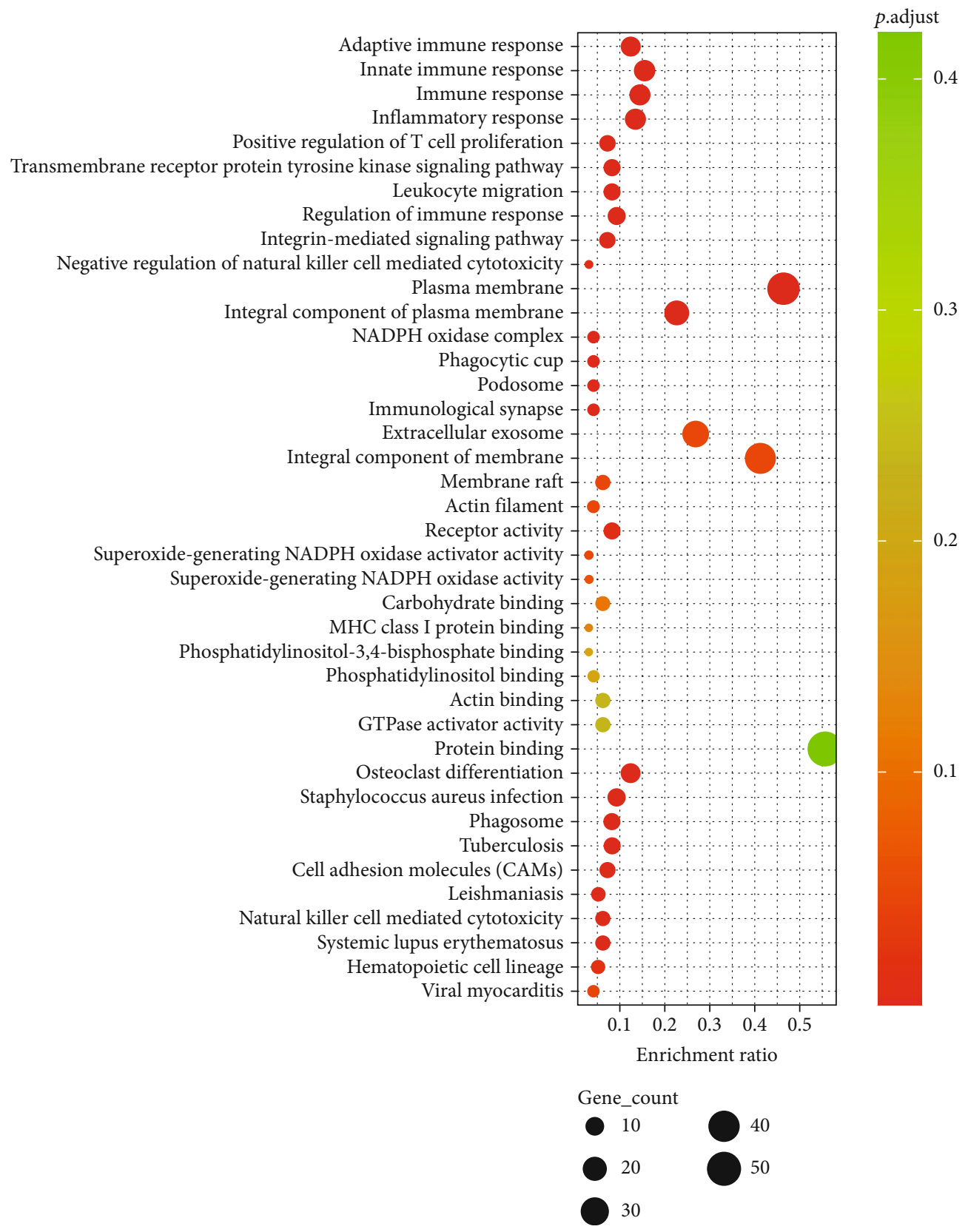

(c)

Figure 8: Enrichment pathway of NCFs in KIRC. (a) PPI network of NCF1, NCF2, and NCF4 (GeneMANIA). Different colors of the network edge indicate the bioinformatics methods applied: physical interactions, coexpression, predicted, colocalization, pathway, genetic interactions, and shared protein domains. (b) PPI network of NCF1, NCF2, and NCF4 (STRING). (c) Bubble diagram showed the GO enrichment in cellular component (CC) terms, biological process (BP) terms, and molecular function (MF) terms and bar plot of Kyoto Encyclopedia of Genes and Genomes (KEGG) enriched terms.

Immune infiltrating cells in the tumor environment have received more and more attention, and many of their functions have been found to be targets for tumor treatment. As early as 1985, Miller et al. began to study the effect of active immunotherapy for renal cell carcinoma [40]. Gillon et al. explored the role of the immune response reflected in the migration inhibition factor (MIF) test in the defense mechanism of RCC patients and explored the role of macrophages in kidney cancer [41].
The results of expression with NCFs and TIMER database results indicate that these three genes are strongly related to SPI1, HCK, VAV1, CD53, ITGB2, and so on. According to the results of KEGG pathway enrichment, they are mainly involved in inflammation and immune response or tumorigenesis [42-46]. Our results show that NCFs and their coexpressed genes, including SPI1, HCK, VAV1, CD53, and ITGB2, are significantly associated with tumorinfiltrating immune cells, especially with immunosuppressive 
TABLE 3: Correlation analysis between NCF1, NCF2, and NCF4 and gene markers of immune cells.

\begin{tabular}{|c|c|c|c|c|c|c|c|c|c|c|c|c|c|}
\hline \multirow{3}{*}{ Description } & \multirow{3}{*}{ Gene markers } & \multicolumn{4}{|c|}{ NCF1 } & \multicolumn{4}{|c|}{ NCF2 } & \multicolumn{4}{|c|}{ NCF4 } \\
\hline & & \multicolumn{2}{|c|}{ None } & \multicolumn{2}{|c|}{ Purity } & \multicolumn{2}{|c|}{ None } & \multicolumn{2}{|c|}{ Purity } & \multicolumn{2}{|c|}{ None } & \multicolumn{2}{|c|}{ Purity } \\
\hline & & Cor & $p$ & Cor & $p$ & Cor & $p$ & Cor & $p$ & Cor & $p$ & Cor & $p$ \\
\hline \multirow{2}{*}{ CD8 T cell } & CD8A & 0.612 & $* * *$ & 0.574 & $* * *$ & 0.436 & $* * *$ & 0.393 & $* * *$ & 0.482 & $* * *$ & 0.403 & $* * *$ \\
\hline & $\mathrm{CD} 8 \mathrm{~B}$ & 0.606 & $* * *$ & 0.572 & $* * *$ & 0.405 & $* * *$ & 0.362 & $* * *$ & 0.488 & $* * *$ & 0.424 & $* * *$ \\
\hline \multirow{3}{*}{ T cell (general) } & CD3D & 0.675 & $* * *$ & 0.642 & $* * *$ & 0.446 & $* * *$ & 0.399 & $* * *$ & 0.601 & $* * *$ & 0.536 & $* * *$ \\
\hline & CD3E & 0.694 & $* * *$ & 0.661 & $* * *$ & 0.462 & $* * *$ & 0.413 & $* * *$ & 0.592 & $* * *$ & 0.527 & $* * *$ \\
\hline & $\mathrm{CD} 2$ & 0.702 & $* * *$ & 0.672 & $* * *$ & 0.510 & $* * *$ & 0.471 & $* * *$ & 0.600 & $* * *$ & 0.538 & $* * *$ \\
\hline \multirow{2}{*}{ B cell } & CD19 & 0.448 & $* * *$ & 0.404 & $* * *$ & 0.282 & $* * *$ & 0.247 & $* * *$ & 0.536 & $* * *$ & 0.492 & $* * *$ \\
\hline & CD79A & 0.503 & $* * *$ & 0.455 & $* * *$ & 0.316 & $* * *$ & 0.265 & $* * *$ & 0.538 & $* * *$ & 0.482 & $* * *$ \\
\hline \multirow{2}{*}{ Monocyte } & CD86 & 0.735 & $* * *$ & 0.715 & $* * *$ & 0.825 & $* * *$ & 0.826 & $* * *$ & 0.704 & $* * *$ & 0.679 & $* * *$ \\
\hline & CD115 (CSF1R) & 0.684 & $* * *$ & 0.665 & $* * *$ & 0.685 & $* * *$ & 0.677 & $* * *$ & 0.738 & $* * *$ & 0.720 & $* * *$ \\
\hline \multirow{3}{*}{ TAM } & CCL2 & 0.129 & $* *$ & 0.087 & 0.062 & 0.182 & $* * *$ & 0.154 & $* *$ & 0.130 & $* *$ & 0.077 & 0.100 \\
\hline & CD68 & 0.545 & $* * *$ & 0.528 & $* * *$ & 0.613 & $* * *$ & 0.618 & $* * *$ & 0.484 & $* * *$ & 0.493 & $* * *$ \\
\hline & IL10 & 0.527 & $* * *$ & 0.469 & $* * *$ & 0.639 & $* * *$ & 0.603 & $* * *$ & 0.600 & $* * *$ & 0.558 & $* * *$ \\
\hline \multirow{3}{*}{ M1 macrophage } & INOS (ISYNA1) & -0.070 & 0.105 & -0.113 & $*$ & -0.198 & $* * *$ & -0.238 & $* * *$ & 0.068 & 0.116 & 0.023 & 0.622 \\
\hline & IRF5 & 0.524 & $* * *$ & 0.522 & $* * *$ & 0.442 & $* * *$ & 0.463 & $* * *$ & 0.406 & $* * *$ & 0.411 & $* * *$ \\
\hline & $\operatorname{cox} 2$ (PTGS2) & -0.015 & 0.733 & -0.057 & 0.219 & 0.108 & $*$ & 0.089 & 0.056 & 0.163 & $* * *$ & 0.117 & $*$ \\
\hline \multirow{3}{*}{ M2 macrophage } & CD163 & 0.483 & $* * *$ & 0.458 & $* * *$ & 0.671 & $* * *$ & 0.664 & $* * *$ & 0.567 & $* * *$ & 0.551 & $* * *$ \\
\hline & VSIG4 & 0.597 & $* * *$ & 0.578 & $* * *$ & 0.636 & $* * *$ & 0.624 & $* * *$ & 0.703 & $* * *$ & 0.686 & $* * *$ \\
\hline & MS4A4A & 0.530 & $* * *$ & 0.496 & $* * *$ & 0.698 & $* * *$ & 0.693 & $* * *$ & 0.646 & $* * *$ & 0.619 & $* * *$ \\
\hline & CD66b (CEACAM8) & 0.039 & 0.374 & 0.045 & 0.335 & 0.113 & $* *$ & 0.136 & $* *$ & 0.074 & 0.087 & 0.105 & $*$ \\
\hline Neutrophils & CD11b (ITGAM) & 0.711 & $* * *$ & 0.690 & $* * *$ & 0.780 & $* * *$ & 0.767 & $* * *$ & 0.657 & $* * *$ & 0.637 & $* * *$ \\
\hline & CCR7 & 0.540 & $* * *$ & 0.496 & $* * *$ & 0.463 & $* * *$ & 0.429 & $* * *$ & 0.579 & $* * *$ & 0.540 & $* * *$ \\
\hline & KIR2DL1 & 0.125 & $* *$ & 0.108 & $*$ & 0.102 & $*$ & 0.053 & 0.260 & 0.096 & $*$ & 0.033 & 0.481 \\
\hline & KIR2DL3 & 0.134 & $* *$ & 0.152 & $* *$ & 0.131 & $* *$ & 0.118 & $*$ & 0.126 & $* *$ & 0.110 & $*$ \\
\hline & KIR2DL4 & 0.273 & $* * *$ & 0.260 & $* * *$ & 0.169 & $* * *$ & 0.123 & $* *$ & 0.282 & $* * *$ & 0.239 & $* * *$ \\
\hline Natural killer cell & KIR2DS4 & 0.075 & 0.082 & 0.063 & 0.175 & 0.038 & 0.386 & -0.005 & 0.914 & 0.093 & $*$ & 0.072 & 0.120 \\
\hline & KIR3DL1 & 0.121 & $* *$ & 0.144 & $* *$ & 0.131 & $* *$ & 0.116 & $*$ & 0.088 & $*$ & 0.075 & 0.107 \\
\hline & KIR3DL2 & 0.184 & $* * *$ & 0.174 & $* * *$ & 0.110 & $*$ & 0.091 & 0.051 & 0.165 & $* * *$ & 0.146 & $* *$ \\
\hline & KIR3DL3 & 0.130 & $* *$ & 0.098 & $*$ & 0.073 & 0.092 & 0.056 & 0.231 & 0.144 & $* *$ & 0.122 & $* *$ \\
\hline & HLA-DPA1 & 0.702 & $* * *$ & 0.672 & $* * *$ & 0.697 & $* * *$ & 0.680 & $* * *$ & 0.576 & $* * *$ & 0.522 & $* * *$ \\
\hline & HLA-DPB1 & 0.763 & $* * *$ & 0.749 & $* * *$ & 0.686 & $* * *$ & 0.667 & $* * *$ & 0.640 & $* * *$ & 0.609 & $* * *$ \\
\hline & HLA-DQB1 & 0.487 & $* * *$ & 0.442 & $* * *$ & 0.419 & $* * *$ & 0.376 & $* * *$ & 0.402 & $* * *$ & 0.341 & $* * *$ \\
\hline Dendritic cell & HLA-DRA & 0.731 & $* * *$ & 0.714 & $* * *$ & 0.750 & $* * *$ & 0.741 & $* * *$ & 0.612 & $* * *$ & 0.583 & $* * *$ \\
\hline & BDCA-1 (CD1C) & 0.395 & $* * *$ & 0.345 & $* * *$ & 0.460 & $* * *$ & 0.419 & $* * *$ & 0.429 & $* * *$ & 0.379 & $* * *$ \\
\hline & BDCA-4 (NRP1) & -0.001 & 0.982 & -0.064 & 0.167 & 0.212 & $* * *$ & 0.166 & $* * *$ & 0.050 & 0.247 & -0.015 & 0.756 \\
\hline & CD11c (ITGAX) & 0.655 & $* * *$ & 0.629 & $* * *$ & 0.676 & $* * *$ & 0.670 & $* * *$ & 0.645 & $* * *$ & 0.639 & $* * *$ \\
\hline & T-bet (TBX21) & 0.455 & $* * *$ & 0.434 & $* * *$ & 0.297 & $* * *$ & 0.261 & $* * *$ & 0.426 & $* * *$ & 0.383 & $* * *$ \\
\hline & STAT4 & 0.454 & $* * *$ & 0.404 & $* * *$ & 0.373 & $* * *$ & 0.342 & $* * *$ & 0.523 & $* * *$ & 0.465 & $* * *$ \\
\hline Th1 & IFN- $\gamma$ (IFNG) & 0.592 & $* * *$ & 0.553 & $* * *$ & 0.410 & $* * *$ & 0.365 & $* * *$ & 0.453 & $* * *$ & 0.382 & $* * *$ \\
\hline & TNF- $\alpha(\mathrm{TNF})$ & 0.478 & $* * *$ & 0.475 & $* * *$ & 0.388 & $* * *$ & 0.384 & $* * *$ & 0.462 & $* * *$ & 0.437 & $* * *$ \\
\hline & STAT1 & 0.588 & $* * *$ & 0.537 & $* * *$ & 0.633 & $* * *$ & 0.605 & $* * *$ & 0.428 & $* * *$ & 0.352 & $* * *$ \\
\hline & GATA3 & 0.212 & $* * *$ & 0.234 & $* * *$ & 0.133 & $* *$ & 0.118 & $*$ & 0.222 & $* * *$ & 0.188 & $* * *$ \\
\hline & STAT6 & 0.150 & $* * *$ & 0.167 & $* * *$ & 0.189 & $* * *$ & 0.206 & $* * *$ & 0.099 & $*$ & 0.138 & $* *$ \\
\hline $\ln 2$ & STAT5A & 0.629 & $* * *$ & 0.614 & $* * *$ & 0.598 & $* * *$ & 0.583 & $* * *$ & 0.535 & $* * *$ & 0.495 & $* * *$ \\
\hline & IL13 & 0.053 & 0.219 & 0.038 & 0.420 & -0.063 & 0.145 & -0.061 & 0.194 & 0.173 & $* * *$ & 0.165 & $* * *$ \\
\hline Tfh & BCL6 & -0.029 & 0.500 & -0.034 & 0.465 & 0.029 & 0.499 & 0.028 & 0.544 & 0.091 & $*$ & 0.098 & $*$ \\
\hline
\end{tabular}



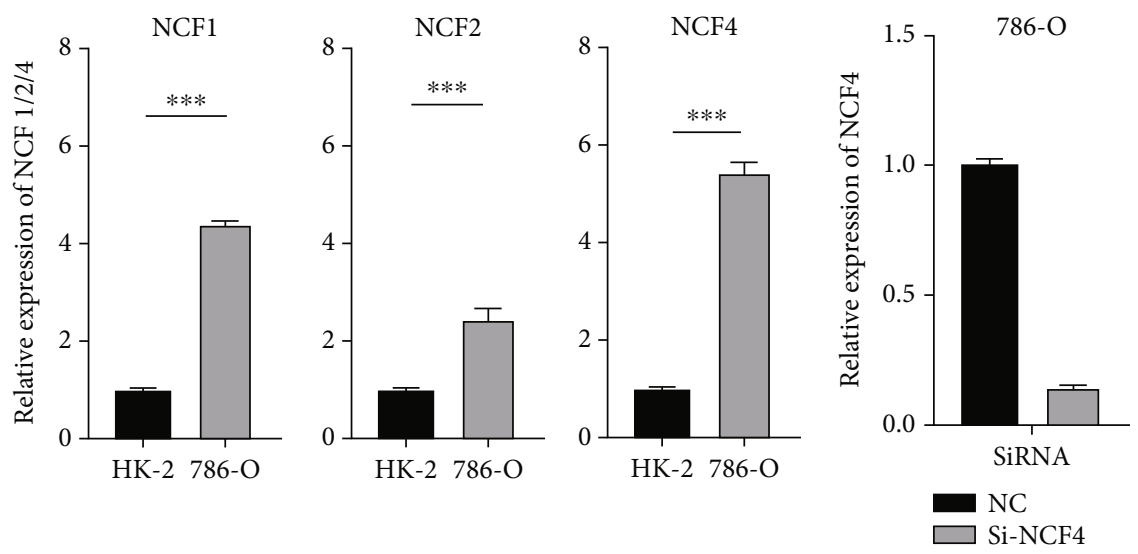

(a)

(b)

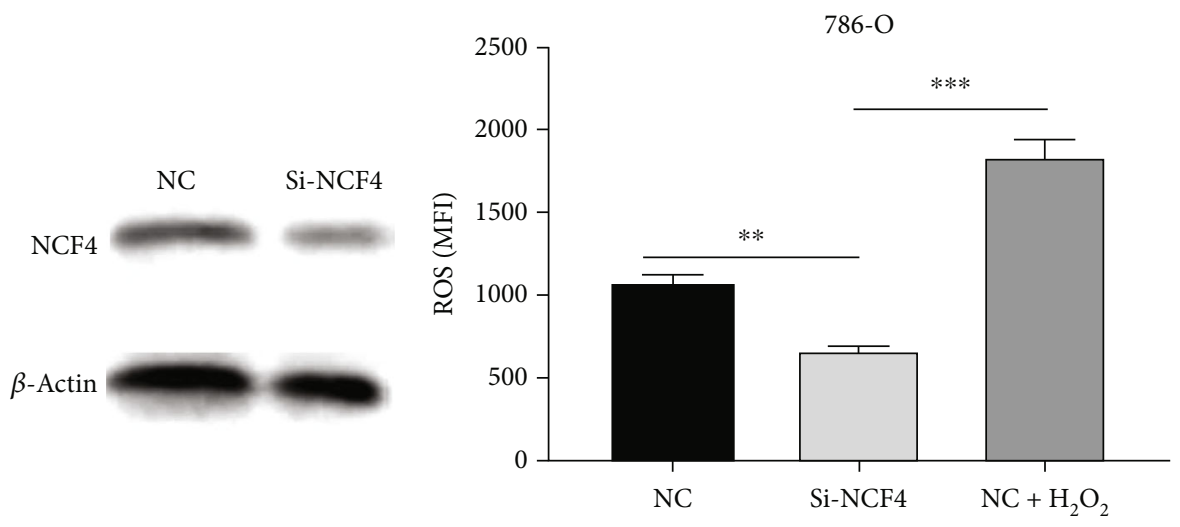

(c)

(d)
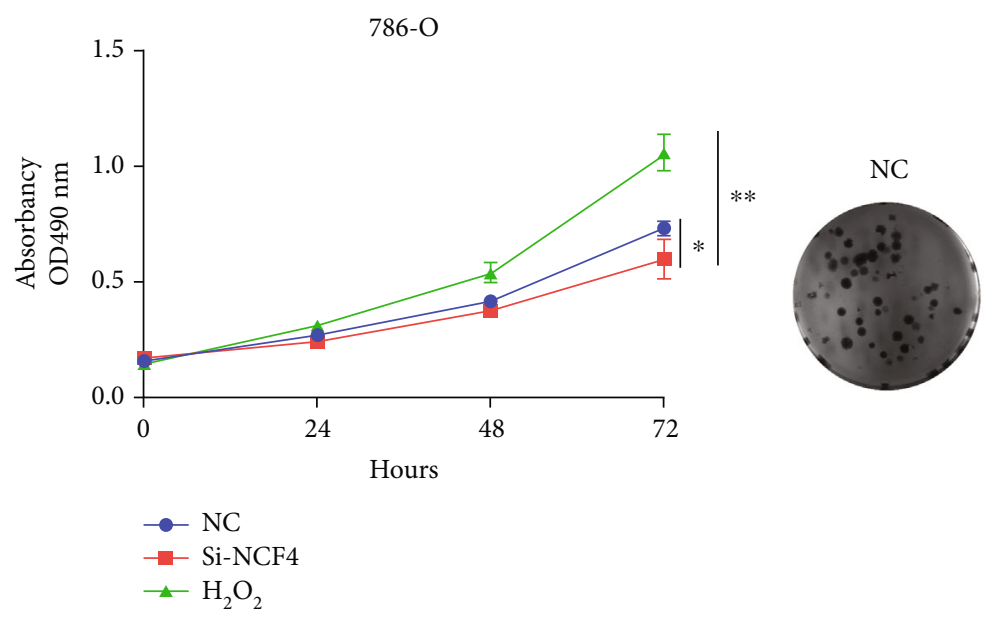

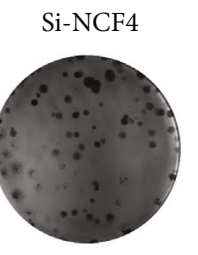

(f)

(e)

Figure 9: NCF4 promotes KIRC cell proliferation. (a) Expression levels of NCF1, NCF2, and NCF4 in HK-2 and 786-O cell lines were determined by qRT-PCR. NCF4 silencing effect was examined by (b) qRT-PCR and (c) western blotting in 786-O cell lines. After nonlethal oxidative stress induced by $500 \mu \mathrm{M} \mathrm{H}_{2} \mathrm{O}_{2}$ for 3 hours to 786-O cells and NCF4 knockdown 786-O cells, (d) flow cytometry was used to evaluate the ROS levels, (e) CCK- 8 assay was performed to measure cell viability, and colony formation assay was used to test cell proliferation. ${ }^{* * *} p<0.001$.

macrophages. According to the result of the TISIDB database, the mRNA levels of NCF1 and NCF4 were obviously decreased in immunologically quiet KIRC immune subtype. According to the difference in copy number, both arm-level deletion and arm-level gain of NCFs may induce changes in the level of immune cell infiltration. These results indicate that the expression level of NCFs is closely related to tumor-infiltrating immune cells. NCFs and their coexpressed 


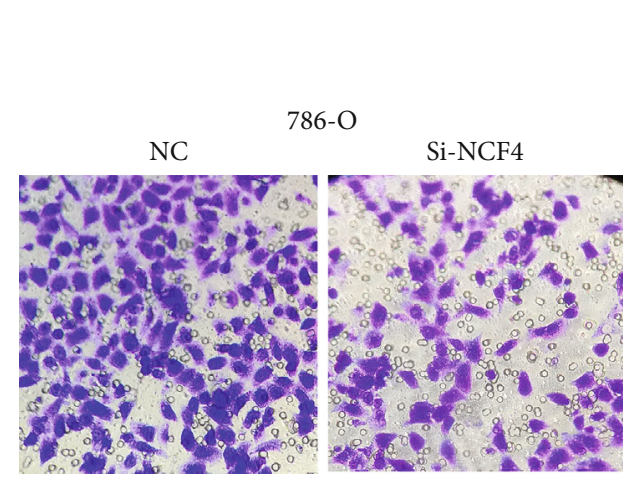

(a)
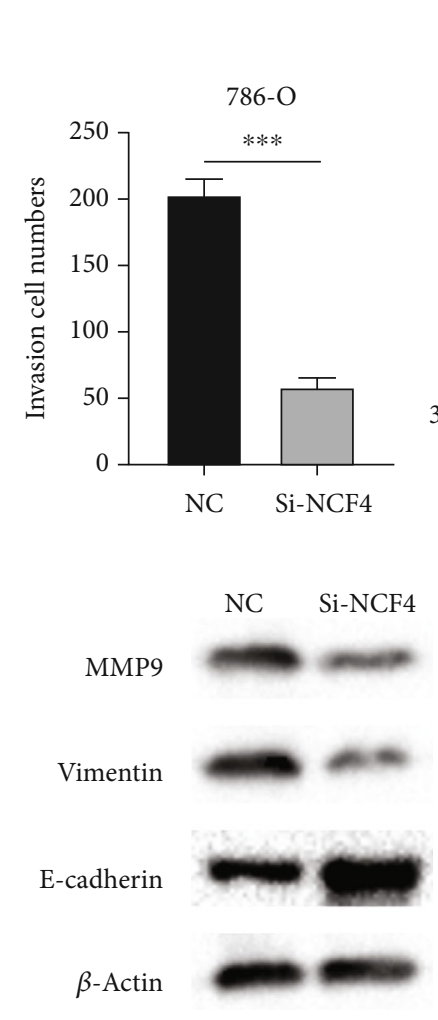

(c)

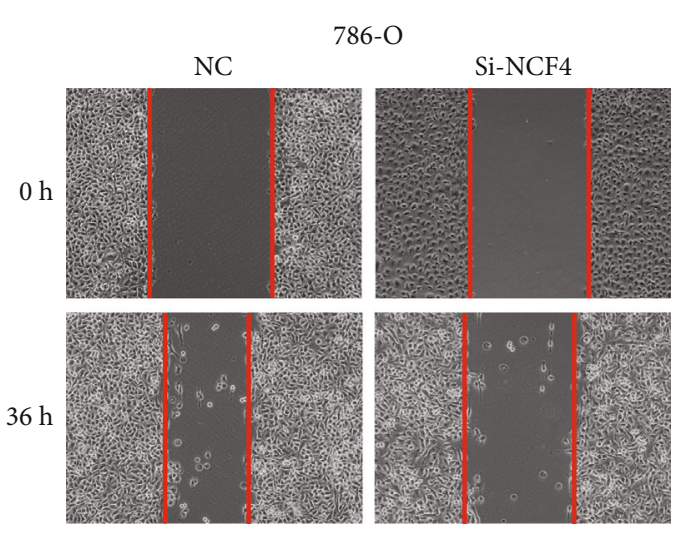

(b)

FIGURE 10: NCF4 promotes KIRC cell epithelial-mesenchymal transition. (a) A transwell assay was applied to detect the effect of NCF4 silencing with respect to the migration of 786-O cells. (b) A wound healing assay was used to detect cell migration of NCF4 knockdown 786-O cells. (c) Effects of NCF4 on the expression levels of EMT-associated proteins in NCF4 knockdown 786-O cells were determined by western blotting. Data represent the mean \pm SD of three separate experiments. ${ }^{* * *} p<0.001$. EMT: epithelial-mesenchymal transition.

genes may participate in the regulation of the immune response of renal cell carcinoma, leading to a poor prognosis of GC patients.

It is worth mentioning that when exploring the markers related to NCFs and immune cells, we found that NCFs showed a strong correlation with M2 macrophages and tumor-associated macrophages, but not with M1 macrophages. The above data indicate that the high expression level of NCFs may promote the differentiation of macrophages into M2 macrophages and finally into TAM, which may contribute to the occurrence of kidney cancer and lead to a poor prognosis. Overexpression of NCFs may induce the massive production of ROS in the tumor immune microenvironment. Studies have shown that TAM induced by ROS can promote tumor proliferation in vitro [47] and angiogenesis [48]. In addition, the sources of ROS production are also different, partly from the tumor cells themselves, but also from other stromal cells in the microenvironment, such as tumor-associated fibroblasts [49] and neutrophils [50].

However, the comprehensive analysis of the NCF family is carried out using different databases and algorithms, and there are still some limitations. First of all, the prognostic NCF family themselves and their related gene characteristics have not been widely adopted and verified. Considering that there are some potential obstacles to the promotion of sequencing technology in clinical tumor detection, the era when molecular prognostic biomarkers are widely used in clinical practice will take more time. Secondly, the role of the NCF family in tumors is only based on our bioinformatics analysis, and the internal mechanism of its impact on tumors deserves more exploration. Thirdly, as a retrospective study, the main limitation of this study lies in its retrospective nature. Therefore, it is necessary to conduct a multicenter prospective study in the future.

\section{Conclusion}

In summary, we found that the overexpression of NCFs in KIRC is associated with clinical manifestations and predicts a poor prognosis. The high expression of NCFs is closely related to the level of infiltrating immune cells, and it may promote the differentiation of macrophages into TAM. Targeting NCFs, NOX2, and ROS-related pathways may become a new antitumor treatment strategy by regulating immune infiltration. These results bring new insights into NCF-mediated KIRC immune regulation.

\section{Data Availability}

The datasets analyzed in the current study are publicly available in cBioPortal and TCGA and accessed via the Genomic Data Commons Data Portal (https://gdc.cancer.gov). The 
original contributions presented in the study are included in the article and within the Supplementary Materials.

\section{Consent}

All the datasets were retrieved from the publishing literature, so it was confirmed that all written informed consent was obtained.

\section{Conflicts of Interest}

There is no conflict of interest between the authors of this article.

\section{Acknowledgments}

This work was supported by the Key Research and Development Program of Shaanxi (grant number 2021ZDLSF02-06).

\section{Supplementary Materials}

Table S1: 100 top coexpression genes with NCFs. NCFs' coexpressed genes (100) were identified from GEPIA2 and conducted pathway enrichment analyses via the DAVID tool. (Supplementary Materials)

\section{References}

[1] R. L. Siegel, K. D. Miller, and A. Jemal, "Cancer statistics, 2019," CA: a Cancer Journal for Clinicians, vol. 69, no. 1, pp. 7-34, 2019.

[2] R. J. Motzer, E. Jonasch, N. Agarwal et al., "Kidney Cancer, Version 2.2017, NCCN Clinical Practice Guidelines in Oncology," Journal of the National Comprehensive Cancer Network, vol. 15, no. 6, pp. 804-834, 2017.

[3] S. Turajlic, C. Swanton, and C. Boshoff, "Kidney cancer: the next decade," The Journal of Experimental Medicine, vol. 215, no. 10, pp. 2477-2479, 2018.

[4] W. M. Linehan, G. Bratslavsky, P. A. Pinto et al., "Molecular diagnosis and therapy of kidney cancer," Annual Review of Medicine, vol. 61, no. 1, pp. 329-343, 2010.

[5] W. M. Linehan, L. S. Schmidt, D. R. Crooks et al., "The metabolic basis of kidney cancer," Cancer Discovery, vol. 9, no. 8, pp. 1006-1021, 2019.

[6] W. M. Linehan, "Genetic basis of kidney cancer: role of genomics for the development of disease-based therapeutics," Genome Research, vol. 22, no. 11, pp. 2089-2100, 2012.

[7] L. Vuong, R. R. Kotecha, M. H. Voss, and A. A. Hakimi, "Tumor microenvironment dynamics in clear-cell renal cell carcinoma," Cancer Discovery, vol. 9, no. 10, pp. 1349-1357, 2019.

[8] E. M. Posadas and R. A. Figlin, "Systemic therapy in renal cell carcinoma: advancing paradigms," Oncology (Williston Park), vol. 26, no. 3, pp. 290-301, 2012.

[9] L. Yin, W. Li, G. Wang et al., "NR1B2 suppress kidney renal clear cell carcinoma (KIRC) progression by regulation of LATS 1/2-YAP signaling," Journal of Experimental \& Clinical Cancer Research, vol. 38, no. 1, p. 343, 2019.

[10] M. W. Kramer, S. Steffens, C. von Klot, A. S. Merseburger, and M. A. Kuczyk, "Systemic therapy for metastatic renal cell carcinoma," Aktuelle Urol, vol. 43, no. 4, pp. 265-268, 2012.
[11] J. Zhong, L. M. Olsson, V. Urbonaviciute, M. Yang, L. Backdahl, and R. Holmdahl, "Association of NOX2 subunits genetic variants with autoimmune diseases," Free Radical Biology \& Medicine, vol. 125, pp. 72-80, 2018.

[12] M. Chiriaco, I. Salfa, G. Di Matteo, P. Rossi, and A. Finocchi, "Chronic granulomatous disease: clinical, molecular, and therapeutic aspects," Pediatric Allergy and Immunology, vol. 27, no. 3, pp. 242-253, 2016.

[13] E. Tarazona-Santos, M. Machado, W. C. Magalhaes et al., "Evolutionary dynamics of the human NADPH oxidase genes CYBB, CYBA, NCF2, and NCF4: functional implications," Molecular Biology and Evolution, vol. 30, no. 9, pp. 21572167, 2013

[14] L. A. Denson, I. Jurickova, R. Karns et al., "Clinical and genomic correlates of neutrophil reactive oxygen species production in pediatric patients with Crohn's disease," Gastroenterology, vol. 154, no. 8, pp. 2097-2110, 2018.

[15] S. Winter, M. Hultqvist Hopkins, F. Laulund, and R. Holmdahl, "A reduction in intracellular reactive oxygen species due to a mutation in NCF4 promotes autoimmune arthritis in mice," Antioxidants \& Redox Signaling, vol. 25, no. 18, pp. 983-996, 2016.

[16] A. Martner, E. Aydin, and K. Hellstrand, "NOX2 in autoimmunity, tumor growth and metastasis," The Journal of Pathology, vol. 247, no. 2, pp. 151-154, 2019.

[17] M. Giefing, S. Winoto-Morbach, J. Sosna et al., "HodgkinReed-Sternberg cells in classical Hodgkin lymphoma show alterations of genes encoding the NADPH oxidase complex and impaired reactive oxygen species synthesis capacity," PLoS One, vol. 8, no. 12, article e84928, 2013.

[18] D. R. Rhodes, J. Yu, K. Shanker et al., "ONCOMINE : a cancer microarray database and integrated data-mining platform," Neoplasia, vol. 6, no. 1, pp. 1-6, 2004.

[19] Z. Tang, B. Kang, C. Li, T. Chen, and Z. Zhang, "GEPIA2: an enhanced web server for large-scale expression profiling and interactive analysis," Nucleic Acids Research, vol. 47, no. W1, pp. W556-W560, 2019.

[20] D. S. Chandrashekar, B. Bashel, S. A. H. Balasubramanya et al., "UALCAN: a portal for facilitating tumor subgroup gene expression and survival analyses," Neoplasia, vol. 19, no. 8, pp. 649-658, 2017.

[21] B. Gyorffy, A. Lanczky, A. C. Eklund et al., "An online survival analysis tool to rapidly assess the effect of 22,277 genes on breast cancer prognosis using microarray data of 1,809 patients," Breast Cancer Research and Treatment, vol. 123, no. 3, pp. 725-731, 2010.

[22] S. V. Vasaikar, P. Straub, J. Wang, and B. Zhang, "LinkedOmics: analyzing multi-omics data within and across 32 cancer types," Nucleic Acids Research, vol. 46, no. D1, pp. D956D963, 2018.

[23] J. Gao, B. A. Aksoy, U. Dogrusoz et al., "Integrative analysis of complex cancer genomics and clinical profiles using the cBioPortal," Science Signaling, vol. 6, no. 269, p. pl1, 2013.

[24] T. Li, J. Fan, B. Wang et al., "TIMER: a web server for comprehensive analysis of tumor-infiltrating immune cells," Cancer Research, vol. 77, no. 21, pp. e108-e110, 2017.

[25] B. Ru, C. N. Wong, Y. Tong et al., "TISIDB: an integrated repository portal for tumor-immune system interactions," Bioinformatics, vol. 35, no. 20, pp. 4200-4202, 2019.

[26] D. Szklarczyk, A. Franceschini, S. Wyder et al., "STRING v10: protein-protein interaction networks, integrated over the tree 
of life," Nucleic Acids Research, vol. 43, no. D1, pp. D447D452, 2015.

[27] D. Warde-Farley, S. L. Donaldson, O. Comes et al., "The GeneMANIA prediction server: biological network integration for gene prioritization and predicting gene function," Nucleic Acids Research, vol. 38, suppl_2, pp. W214-W220, 2010.

[28] D. W. Huang, B. T. Sherman, Q. Tan et al., "The DAVID Gene Functional Classification Tool: a novel biological modulecentric algorithm to functionally analyze large gene lists," Genome Biology, vol. 8, no. 9, p. R183, 2007.

[29] G. Y. Liou and P. Storz, "Reactive oxygen species in cancer," Free Radical Research, vol. 44, no. 5, pp. 479-496, 2010.

[30] B. Bakir, A. M. Chiarella, J. R. Pitarresi, and A. K. Rustgi, "EMT, MET, plasticity, and tumor metastasis," Trends in Cell Biology, vol. 30, no. 10, pp. 764-776, 2020.

[31] J. Jones, J. Bhatt, J. Avery et al., “The kidney cancer research priority-setting partnership: identifying the top 10 research priorities as defined by patients, caregivers, and expert clinicians," Cuaj-Canadian Urological Association, vol. 11, no. 12, pp. 379-387, 2017.

[32] A. Znaor, J. Lortet-Tieulent, M. Laversanne, A. Jemal, and F. Bray, "International variations and trends in renal cell carcinoma incidence and mortality," European Urology, vol. 67, no. 3, pp. 519-530, 2015.

[33] J. Usher-Smith, R. K. Simmons, S. H. Rossi, and G. D. Stewart, "Current evidence on screening for renal cancer," Nature Reviews. Urology, vol. 17, no. 11, pp. 637-642, 2020.

[34] C. M. Diaz-Montero, B. I. Rini, and J. H. Finke, "The immunology of renal cell carcinoma," Nature Reviews. Nephrology, vol. 16, no. 12, pp. 721-735, 2020.

[35] S. Ostrand-Rosenberg and C. Fenselau, "Myeloid-derived suppressor cells: immune-suppressive cells that impair antitumor immunity and are sculpted by their environment," Journal of Immunology, vol. 200, no. 2, pp. 422-431, 2018.

[36] S. Galadari, A. Rahman, S. Pallichankandy, and F. Thayyullathil, "Reactive oxygen species and cancer paradox: to promote or to suppress?," Free Radical Biology \& Medicine, vol. 104, pp. 144-164, 2017.

[37] L. van der Weyden, A. O. Speak, A. Swiatkowska et al., "Pulmonary metastatic colonisation and granulomas in NOX2deficient mice," The Journal of Pathology, vol. 246, no. 3, pp. 300-310, 2018.

[38] T. Kelkka, A. Pizzolla, J. P. Laurila et al., "Mice lacking NCF1 exhibit reduced growth of implanted melanoma and carcinoma tumors," PLoS One, vol. 8, no. 12, article e84148, 2013.

[39] W. Tan, M. A. Hildebrandt, X. Pu et al., "Role of inflammatory related gene expression in clear cell renal cell carcinoma development and clinical outcomes," The Journal of Urology, vol. 186, no. 5, pp. 2071-2077, 2011.

[40] G. A. Miller, J. E. Pontes, R. P. Huben, and M. H. Goldrosen, "Humoral immune response of patients receiving specific active immunotherapy for renal cell carcinoma," Cancer Research, vol. 45, no. 9, pp. 4478-4482, 1985.

[41] G. Gillon, E. Livni, and C. Servadio, "Sequential study of macrophage migration inhibition factor in renal cell carcinoma patients," European Urology, vol. 14, no. 5, pp. 391-394, 2017.

[42] H. Zhu, L. Zhang, Y. Wu et al., "T-ALL leukemia stem cell 'stemness' is epigenetically controlled by the master regulator SPI1,” eLife, vol. 7, 2018.
[43] X. Kong, Y. Liao, L. Zhou et al., "Hematopoietic cell kinase (HCK) is essential for NLRP3 inflammasome activation and lipopolysaccharide-induced inflammatory response in vivo," Frontiers in Pharmacology, vol. 11, p. 581011, 2020.

[44] S. Grassilli, F. Brugnoli, R. Lattanzio, S. Buglioni, and V. Bertagnolo, "Vav 1 down-modulates Akt2 expression in cells from pancreatic ductal adenocarcinoma: nuclear Vav1 as a potential regulator of Akt related malignancy in pancreatic cancer," Biomedicine, vol. 8, no. 10, p. 379, 2020.

[45] Z. J. Greenberg, D. A. Monlish, R. L. Bartnett et al., "The tetraspanin CD53 regulates early B cell development by promoting IL-7R signaling," Journal of Immunology, vol. 204, no. 1, pp. 58-67, 2020.

[46] N. Dashti, M. Mahmoudi, F. Gharibdoost et al., "Evaluation of ITGB2 (CD18) and SELL (CD62L) genes expression and methylation of ITGB2 promoter region in patients with systemic sclerosis," Rheumatology International, vol. 38, no. 3, pp. 489-498, 2018.

[47] J. Zhang, H. Li, Q. Wu et al., “Tumoral NOX4 recruits M2 tumor-associated macrophages via ROS/PI3K signalingdependent various cytokine production to promote NSCLC growth," Redox Biology, vol. 22, p. 101116, 2019.

[48] F. Rezende, F. Moll, M. Walter et al., "The NADPH organizers NoxO1 and p47phox are both mediators of diabetes-induced vascular dysfunction in mice," Redox Biology, vol. 15, pp. 1221, 2018.

[49] A. Zhang, Y. Qian, Z. Ye et al., "Cancer-associated fibroblasts promote M2 polarization of macrophages in pancreatic ductal adenocarcinoma," Cancer Medicine, vol. 6, no. 2, pp. 463-470, 2017.

[50] W. Yang, Y. Tao, Y. Wu et al., "Neutrophils promote the development of reparative macrophages mediated by ROS to orchestrate liver repair," Nature Communications, vol. 10, no. 1, p. 1076, 2019. 